\title{
Weak essentially undecidable theories of concatenation
}

\author{
Juvenal Murwanashyaka ${ }^{1}$
}

Received: 1 December 2019 / Accepted: 7 February 2022 / Published online: 7 March 2022

(c) The Author(s) 2022

\begin{abstract}
In the language $\{0,1, \circ, \preceq\}$, where 0 and 1 are constant symbols, $\circ$ is a binary function symbol and $\preceq$ is a binary relation symbol, we formulate two theories, WD and D, that are mutually interpretable with the theory of arithmetic $\mathrm{R}$ and Robinson arithmetic $\mathrm{Q}$, respectively. The intended model of WD and $\mathrm{D}$ is the free semigroup generated by $\{\mathbf{0}, \mathbf{1}\}$ under string concatenation extended with the prefix relation. The theories WD and $\mathrm{D}$ are purely universally axiomatised, in contrast to $\mathrm{Q}$ which has the $\Pi_{2}$-axiom $\forall x[x=0 \vee \exists y[x=S y]]$.
\end{abstract}

Keywords Theory of concatenation · First-order arithmetic · Interpretability

Mathematics Subject Classification 03B25 -03B30 - 03F25 -03F30 - 03F40

\section{Introduction}

This paper follows the line of work that focuses on determining whether there is a weakest theory that is essentially undecidable. We formulate two natural essentially undecidable theories in the language of concatenation that are purely universally axiomatised.

A countable first-order theory is called essentially undecidable if any consistent extension, in the same language, is undecidable (there is no algorithm for deciding whether an arbitrary sentence is a theorem). A countable first-order theory is called essentially incomplete if any recursively axiomatizable consistent extension is incomplete. It is known that a theory is essentially undecidable if and only if it is essentially incomplete. Indeed, if a theory is not essentially undecidable, then by definition it has a consistent decidable extension which can be extended to a decidable complete consistent theory (see Chapter 1 of Tarski et al. [18]). On the other hand, if a theory is not essentially incomplete, then by definition it has a complete consistent recursively

\footnotetext{
$凶 \quad$ Juvenal Murwanashyaka juvenalm@math.uio.no

1 Department of Mathematics, University of Oslo, Oslo, Norway
} 
axiomatizable extension which clearly is decidable. Two theories that are known to be essentially undecidable are Robinson arithmetic Q and the related theory R. Rosser's generalization of Gödel's first incompleteness theorem is usually taken as the statement that $\mathrm{Q}$ is essentially undecidable.

\section{The Axioms of $\mathrm{R}$}

$$
\begin{aligned}
& \mathrm{R}_{1} \bar{n}+\bar{m}=\overline{n+m} \\
& \mathrm{R}_{2} \bar{n} \times \bar{m}=\overline{n \times m} \\
& \mathrm{R}_{3} \bar{n} \neq \bar{m} \\
& \mathrm{R}_{4} \forall x\left[x \leq \bar{n} \rightarrow \bigvee_{k \leq n} x=\bar{k}\right] \\
& \mathrm{R}_{5} \forall x[x \leq \bar{n} \vee \bar{n} \leq \bar{x}]
\end{aligned}
$$

\section{The Axioms of $\mathrm{Q}$}

The main objective of this paper is to show that two theories, WD and D, are mutually interpretable with $R$ and $Q$, respectively. The axioms of WD and $D$ are given below using juxtaposition instead of the binary function symbol of the formal language. Later we regard $D$ as the theory without the axiom $D_{5}$. We do this because the two versions of $D$ are mutually interpretable. The theories WD and $D$ are theories in the language of concatenation extended with a binary relation symbol. That is, the language $\{0,1, \circ, \preceq\}$ where 0 and 1 are constant symbols, $\circ$ is a binary function symbol and $\preceq$ is a binary relation symbol. The intended structure $\mathfrak{D}$ is the free semigroup with two generators extended with the prefix relation which we denote $\preceq^{\mathfrak{D}}$. As in number theory, each element $\alpha$ in the universe is associated with a canonical term $\bar{\alpha}$. The inclusion of a binary relation in the languages makes it easier to define $\Sigma_{1}$-formulas and to give a purely universal $\Sigma_{1}$-complete axiomatization of $\mathfrak{D}$. We observe that any theory that proves all true $\Sigma_{1}$-sentences is an extension of WD. Indeed, instances of WD $\mathrm{W}_{1}$ and WD are true $\Sigma_{1}$-sentences, and each instance of $W_{3}$ follows from the true $\Sigma_{1}$-sentences $\forall x \preceq \bar{\alpha}\left[\bigvee_{\gamma \preceq^{\mathcal{D}} \alpha} x=\bar{\gamma}\right]$ and $\bigwedge_{\gamma \preceq^{\mathcal{D}} \alpha} \bar{\gamma} \preceq \bar{\alpha}$. The theory WD is thus the weakest $\Sigma_{1}$-complete axiomatization of $\mathfrak{D}$ (modulo closure under logical implication) and the theory $\mathrm{D}$ is a natural finitely axiomatizable extension of WD. A variant of $\mathrm{D}$ where we have an identity element was introduced in Kristiansen and Murwanashyaka [9] as a $\Sigma_{1}$-complete axiomatization of the structure $\mathfrak{D}$ extended with the empty string. In [9, $10]$, we identify a number of decidable and undecidable fragments of $\mathfrak{D}$ and related structures.

The Axioms of WD

$$
\begin{array}{ll}
\mathrm{WD}_{1} \bar{\alpha} \bar{\beta}=\overline{\alpha \beta} & \mathrm{D}_{1} \forall x y z[(x y) z=x(y z)] \\
\mathrm{WD}_{2} \bar{\alpha} \neq \bar{\beta} \quad \text { if } \alpha \neq \beta & \mathrm{D}_{2} \forall x y[x \neq y \rightarrow(x 0 \neq y 0 \wedge x 1 \neq y 1)] \\
\mathrm{WD}_{3} \forall x\left[x \preceq \bar{\alpha} \leftrightarrow \bigvee_{\gamma \preceq^{\mathfrak{D}_{\alpha}}} x=\bar{\gamma}\right] & \mathrm{D}_{3} \forall x y[x 0 \neq y 1] \\
& \mathrm{D}_{4} \forall x[x \preceq 0 \leftrightarrow x=0] \\
& \mathrm{D}_{5} \forall x[x \preceq 1 \leftrightarrow x=1] \\
& \mathrm{D}_{6} \forall x y[x \preceq y 0 \leftrightarrow(x=y 0 \vee x \preceq y)] \\
& \mathrm{D}_{7} \forall x y[x \preceq y 1 \leftrightarrow(x=y 1 \vee x \preceq y)]
\end{array}
$$


The theory of concatenation TC was introduced by Grzegorczyk in [4] where he also showed that it is undecidable. The language of TC consists only of the two constant symbols 0 and 1 and the binary function symbol $\circ$. The intended model of TC is a free semigroup with at least two generators. That is, a structure of the form $\left(\Gamma^{+}, a_{1}, \ldots, a_{n}, \frown\right)$ where $\Gamma=\left\{a_{1}, \ldots, a_{n}\right\}$ is a finite alphabet with at least two symbols, $\Gamma^{+}$is the set of all finite non-empty strings over $\Gamma$ and $\frown$ is the binary operator that concatenates elements of $\Gamma^{+}$. Grzegorczyk's motivation for introducing the theory TC was that, as computation involves manipulation of text, the notion of computation can be formulated on the basis of discernibility of text without reference to natural numbers. Then, undecidability of first-order logic and essential undecidability can be explained using a theory of strings thereby avoiding complicated coding of syntax based on natural numbers. In [5], Grzegorczyk and Zdanowski showed that TC is essentially undecidable. This was further improved in Ganea [3], Visser [19] and Švejdar [16] where it was shown that TC is mutually interpretable with Robinson arithmetic. In Higuchi and Horihata [6], it was shown that TC is minimal essentially undecidable. That is, removing any one of the axioms of TC gives a theory that is not essentially undecidable. We will refer to $\mathrm{TC}$ as $\mathrm{TC}^{-\varepsilon}$ and we will let $\mathrm{TC}$ refer to the theory where we have an identity element. The two theories are known to be mutually interpretable (see Grzegorczyk and Zdanowski [5] and Visser [19]). In the article [6], it was also shown that a weak theory of concatenation $\mathrm{WTC}^{-\varepsilon}$ is minimal essentially undecidable and mutually interpretable with $\mathrm{R}$. That $\mathrm{WTC}^{-\varepsilon}$ is minimal essentially undecidable means that removal of any one of the axiom schemas of WTC ${ }^{-\varepsilon}$ gives a theory that is not essentially undecidable.

\section{The Axioms of $\mathrm{TC}^{-\varepsilon}$}

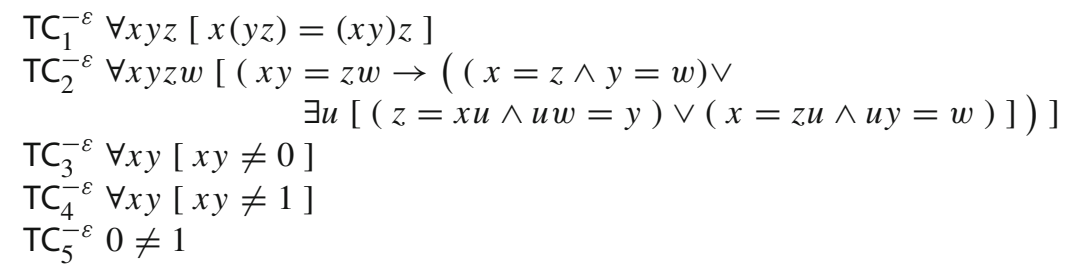

\section{The Axioms of WTC ${ }^{-\varepsilon}$}

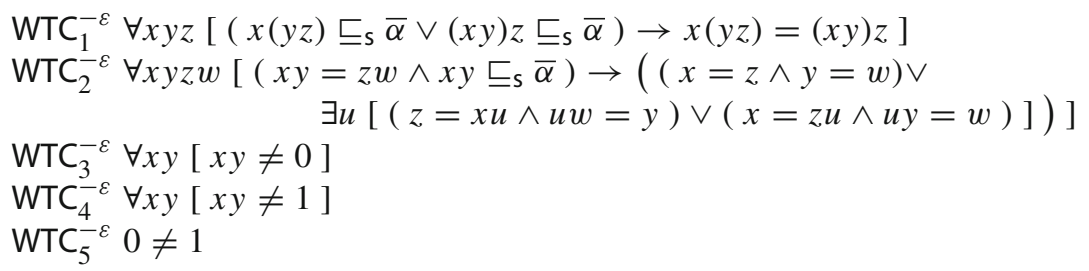

We use $x \sqsubseteq$ s $y$ as shorthand for

$$
y=x \vee \exists u v[y=u x \vee y=x v \vee y=(u x) v \vee y=u(x v)] .
$$


Fig. $1 S \rightarrow T$ means $S$ is interpretable in $T$ but $S$ does not interpret $T . S \leftrightarrow T$ means $S$ and $T$ are mutually interpretable

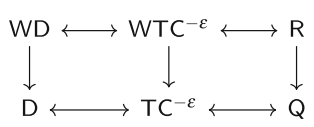

The diagram in Fig. 1 summarizes the relationships between R, Q, WD, D, WTC ${ }^{-\varepsilon}$ and $\mathrm{TC}^{-\varepsilon}$.

Kristiansen and Murwanashyaka [11] have also introduced two essentially undecidable theories, WT and T, that are purely universally axiomatised. While WD and D are theories of concatenation, WT and T are theories of terms. The intended model of WT and $T$ is the extended term algebra given by the language $\mathscr{L}_{T}=\{\perp,\langle\cdot, \cdot\rangle$, ᄃ $\}$ where $\perp$ is a constant symbol, $\langle\cdot, \cdot\rangle$ is a binary function symbol and $\sqsubseteq$ is a binary relation symbol. The universe is the set of all variable-free $\mathscr{L}_{T}$-terms and $\langle\cdot, \cdot\rangle$ is interpreted as the function that maps a pair $(s, t)$ to the term $\langle s, t\rangle$. Every variable-free term is thus realized as itself. The relation symbol $\sqsubseteq$ is interpreted as the subterm relation. The theory WT has a compact axiomatization that consists only of analogues of $\mathrm{WD}_{2}$ and $W D_{3}$. An analogue of $W D_{1}$ is not necessary since it holds by pure logic. The theory $T$ has two axioms

$\mathrm{T}_{1} \equiv \forall x y[\langle x, y\rangle \neq \perp]$ and $\mathrm{T}_{2} \equiv \forall x y z w[\langle x, y\rangle=\langle z, w\rangle \rightarrow(x=z \wedge y=w)]$

that describe $\langle\cdot, \cdot\rangle$; and two axioms

$$
\begin{aligned}
& \mathrm{T}_{3} \equiv \forall x[x \sqsubseteq \perp \leftrightarrow x=\perp] \text { and } \\
& \mathrm{T}_{4} \equiv \forall x y z[x \sqsubseteq\langle y, z\rangle \leftrightarrow(x=\langle y, z\rangle \vee x \sqsubseteq y \vee x \sqsubseteq z)]
\end{aligned}
$$

that describe $\sqsubseteq$. Kristiansen and Murwanashyaka give interpretations of R and Q in WT and T, respectively. A result of Visser [20] ensures that WT is interpretable in R since it is locally finitely satisfiable. They conjecture that $T$ and $Q$ are mutually interpretable.

\section{Preliminaries}

In this section, we clarify a number of notions that we only glossed over in the previous section. We also introduce a number of intermediate theories that will be useful in showing that $W D$ and $R$ are mutually interpretable and that $D$ and $Q$ are mutually interpretable.

We consider the structures

$$
\mathfrak{D}^{-}=\left(\{\mathbf{0}, \mathbf{1}\}^{+}, \mathbf{0}, \mathbf{1}, \frown\right) \text { and } \mathfrak{D}=\left(\{\mathbf{0}, \mathbf{1}\}^{+}, \mathbf{0}, \mathbf{1}, \frown, \preceq^{\mathfrak{D}}\right)
$$

where $\{\mathbf{0}, \mathbf{1}\}^{+}$is the set of all finite non-empty strings over the alphabet $\{\mathbf{0}, \mathbf{1}\}$, the binary operator - concatenates elements of $\{\mathbf{0}, \mathbf{1}\}^{+}$and $\preceq^{\mathcal{D}}$ denotes the prefix relation, i.e., $x \preceq^{\mathfrak{D}} y$ if and only if $y=x$ or there exists $z \in\{\mathbf{0}, \mathbf{1}\}^{+}$such that $y=x^{\frown} z$. The structure $\mathfrak{D}^{-}$is thus the free semigroup with two generators. We call elements of $\{\mathbf{0}, \mathbf{1}\}^{+}$bit strings. The structures $\mathfrak{D}^{-}$and $\mathfrak{D}$ are first-order structures over the languages $\mathscr{L}_{\mathrm{BT}}^{-}=\{0,1, \circ\}$ and $\mathscr{L}_{\mathrm{BT}}=\{0,1, \circ, \preceq\}$, respectively. 
In first-order number theory, each natural number $n$ is associated with a numeral $\bar{n}$. Each non-empty bit string $\alpha \in\{\mathbf{0}, \mathbf{1}\}^{+}$is associated by recursion with a unique $\mathscr{L}_{\mathrm{BT}^{-}}^{-}$ term $\bar{\alpha}$, called a biteral, as follows: $\overline{\mathbf{0}} \equiv 0, \overline{\mathbf{1}} \equiv 1, \overline{\alpha \mathbf{0}} \equiv(\bar{\alpha} \circ 0)$ and $\overline{\alpha \mathbf{1}} \equiv(\bar{\alpha} \circ 1)$. The biterals are important if we, for example, want to show that certain sets are definable since we then need to talk about elements of $\{\mathbf{0}, \mathbf{1}\}^{+}$in the formal theory.

In the language $\mathscr{L}_{\mathrm{BT}}$, we can define the $\Sigma_{1}$-formulas as follows: atomic formulas and their negations are $\Sigma_{1}$-formulas. If $\alpha$ and $\beta$ are $\Sigma_{1}$-formulas, then $\alpha \wedge \beta, \alpha \vee \beta$ and $\exists x \alpha$ are $\Sigma_{1}$-formulas. Furthermore, if the variable $x$ does not occur in the term $t$, then $\exists x[x \preceq t \wedge \alpha]$ and $\forall x[x \preceq t \rightarrow \alpha]$ are also $\Sigma_{1}$-formulas. We use $\exists x \preceq t \alpha$ and $\forall x \preceq t \alpha$ as abbreviations for $\exists x[x \preceq t \wedge \alpha]$ and $\forall x[x \preceq t \rightarrow \alpha]$, respectively. To define $\Sigma_{1}$-formulas over the language $\mathscr{L}_{\mathrm{BT}}^{-}$, we first need to define a binary relation in order to have bounded quantifiers. Two natural choices are

$$
\begin{aligned}
& -x \preceq_{\mathrm{i}} y \equiv y=x \vee \exists z[y=x z] \\
& -x \sqsubseteq_{\mathrm{s}} y \equiv y=x \vee \exists u v[y=u x \vee y=x v \vee y=(u x) v \vee y=u(x v)] .
\end{aligned}
$$

Over the structure $\mathfrak{D}^{-}$, the defined relation $\preceq_{i}$ is realized as the prefix relation while $\sqsubseteq_{\mathrm{s}}$ is realized as the substring relation. Given a bit string $\alpha$, the set of those bit strings that are substrings of $\alpha$ is denoted $\langle\ldots \alpha\rangle$ and consists of those bit strings $\beta$ such that $\beta=\alpha$ or there exist bit strings $u$ and $v$ such that $\alpha=u \beta$ or $\alpha=\beta v$ or $\alpha=u \beta v$. We choose to work with $\sqsubseteq_{s}$ since the intended interpretation of $\preceq$ is the prefix relation. We observe that the number of substrings of a string $\alpha$ is quadratic in the length of $\alpha$, whereas the number of prefixes of $\alpha$ is linear in the length of $\alpha$. This means that the choice between $\preceq_{i}$ and $\sqsubseteq_{s}$ could make a difference in the context of very weak theories.

Having introduced $\Sigma_{1}$-formulas, it is natural to try to find $\Sigma_{1}$-complete axiomatizations of the structures $\mathfrak{D}^{-}$and $\mathfrak{D}$. That is, to find theories that prove all true $\Sigma_{1}$-sentences (sentences are formulas without free variables) and that are such that the non-logical axioms are true over the intended structure. A natural first step is to introduce the theories WBT and WD defined below. It is not difficult to see that these two theories are $\Sigma_{1}$-complete. The theories WBT and WD are not finitely axiomatizable but they are the weakest possible $\Sigma_{1}$-complete axiomatizations of $\mathfrak{D}^{-}$and $\mathfrak{D}$, respectively, modulo closure under logical implication. Once we have WBT and WD, the theories BTQ and $\mathrm{D}+\forall x[x \preceq 1 \leftrightarrow x=1]$ defined below are natural finitely axiomatizable extensions ( $T$ is an extension of $S$ if the language of $S$ is a subset of the language of $T$ and every theorem of $S$ is a theorem of $T$ ). The reason for not having $\forall x[x \preceq 1 \leftrightarrow x=1]$ as an axiom of $\mathrm{D}$ is that it is not necessary for essential undecidability (we could, of course, very well have worked with the theory where we have $\forall x[x \preceq 1 \leftrightarrow x=1]$ instead of $\forall x[x \preceq 0 \leftrightarrow x=0])$. Although the theories WBT, BTQ, WD and D are $\Sigma_{1}$-complete, it is not at all obvious that they are essentially undecidable. When proving that $\mathrm{R}$ is essentially undecidable, the axiom schema $\mathrm{R}_{5} \equiv \forall x[x \leq \bar{n} \vee \bar{n} \leq x]$ is essential. It is however not straightforward to define a binary relation that provably satisfies the analogue of $R_{5}$. The method of relative interpretability then becomes important for establishing that these theories are 
Fig. $2 S \rightarrow T$ means $S$ is interpretable in $T$ but $S$ does not interpret $T . S \leftrightarrow T$ means $S$ and $T$ are mutually interpretable. $S \rightarrow T$ means $S$ is interpretable in $T$ but it is unknown whether $S$ interprets $T$

essentially undecidable.

\section{The Axioms of WBT}

$$
\begin{aligned}
& \mathrm{WBT}_{1} \bar{\alpha} \bar{\beta}=\overline{\alpha \beta} \\
& \mathrm{WBT}_{2} \bar{\alpha} \neq \bar{\beta} \quad \text { if } \alpha \neq \beta \\
& \mathrm{WBT}_{3} \forall x\left[x \sqsubseteq_{\mathrm{s}} \bar{\alpha} \rightarrow \bigvee_{\gamma \in\langle\ldots \alpha\rangle} x=\bar{\gamma}\right]
\end{aligned}
$$

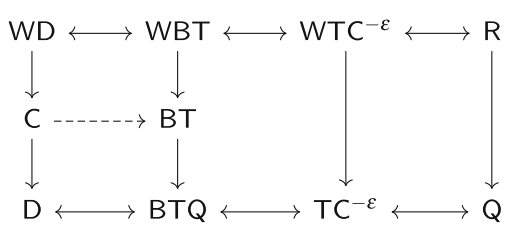

\section{The Axioms of WD}

$\mathrm{WD}_{1} \bar{\alpha} \bar{\beta}=\overline{\alpha \beta}$

$\mathrm{WD}_{2} \bar{\alpha} \neq \bar{\beta} \quad$ if $\alpha \neq \beta$

$\mathrm{WD}_{3} \forall x\left[x \preceq \bar{\alpha} \leftrightarrow \bigvee_{\gamma \leq^{\mathcal{D}} \alpha} x=\bar{\gamma}\right]$

\section{The Axioms of BTQ}

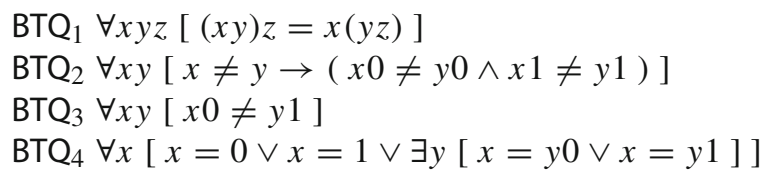

\section{The Axioms of $D$}

$\mathrm{D}_{1} \forall x y z[(x y) z=x(y z)]$

$\mathrm{D}_{2} \forall x y[x \neq y \rightarrow(x 0 \neq y 0 \wedge x 1 \neq y 1)]$

$\mathrm{D}_{3} \forall x y[x 0 \neq y 1]$

$\mathrm{D}_{4} \forall x[x \preceq 0 \leftrightarrow x=0]$

$\mathrm{D}_{5} \forall x y[x \preceq y 0 \leftrightarrow(x=y 0 \vee x \preceq y)]$

$\mathrm{D}_{6} \forall x y[x \preceq y 1 \leftrightarrow(x=y 1 \vee x \preceq y)]$

\section{The Axioms of BT}

The Axioms of $C$

$\mathrm{BTQ}_{1}, \mathrm{BTQ}_{2}, \mathrm{BTQ}_{3}, \mathrm{WBT}_{3} \mathrm{D}_{1}, \mathrm{D}_{2}, \mathrm{D}_{3}, \mathrm{WD}_{3}$

The overall relationship among the various theories introduced so far is summarized in Fig. 2. The constants 0 and 1 are atoms in BTQ. This means that BTQ $\vdash \forall x y[x y \neq$ $0] \wedge \forall x y[x y \neq 1]$ (see Lemma 8 in Ganea [3] for a proof). This observation is used in the proof of Theorem 7 where we show that $D$ is interpretable in BTQ.

We recall the method of relative interpretability introduced by Tarski [18] for showing that first-order theories are essentially undecidable. Let $\mathscr{L}_{1}$ and $\mathscr{L}_{2}$ be computable first-order languages. A relative translation $\tau$ from $\mathscr{L}_{1}$ to $\mathscr{L}_{2}$ is a computable map given by:

1. An $\mathscr{L}_{2}$-formula $\delta(x)$ with exactly one free variable. The formula $\delta(x)$ is called a domain.

2. For each $n$-ary relation symbol $R$ of $\mathscr{L}_{1}$, an $\mathscr{L}_{2}$-formula $\psi_{R}\left(x_{1}, \ldots, x_{n}\right)$ with exactly $n$ free variables. The equality symbol $=$ is treated as a binary relation symbol. 
3. For each $n$-ary function symbol $f$ of $\mathscr{L}_{1}$, an $\mathscr{L}_{2}$-formula $\psi_{f}\left(x_{1}, \ldots, x_{n}, y\right)$ with exactly $n+1$ free variables.

4. For each constant symbol $c$ of $\mathscr{L}_{1}$, an $\mathscr{L}_{2}$-formula $\psi_{c}(y)$ with exactly one free variable.

We extend $\tau$ to a translation of atomic $\mathscr{L}_{1}$-formulas by mapping a $\mathscr{L}_{1}$-term $t$ to a $\mathscr{L}_{2}$-formula $(t)^{\tau, w}$ with a free variable $w$ that denotes the value of $t$ :

5. For each $n$-ary relation symbol $R$ of $\mathscr{L}_{1}$

$$
\left(R\left(t_{1}, \ldots, t_{n}\right)\right)^{\tau} \equiv \exists v_{1} \ldots v_{n}\left[\bigwedge_{i=1}^{n} \delta\left(v_{i}\right) \wedge \bigwedge_{j=1}^{n}\left(t_{j}\right)^{\tau, v_{j}} \wedge \psi_{R}\left(v_{1}, \ldots, v_{n}\right)\right]
$$

where $v_{1}, \ldots, v_{n}$ are distinct variable symbols that do not occur in $t_{1}, \ldots, t_{n}$ and

(a) for each variable symbol $x$ of $\mathscr{L}_{1},(x)^{\tau, w} \equiv w=x$

(b) for each constant symbol $c$ of $\mathscr{L}_{1},(c)^{\tau, w} \equiv \psi_{c}(w)$

(c) for each $n$-ary function symbol $f$ of $\mathscr{L}_{1}$

$$
\left(f\left(t_{1}, \ldots, t_{n}\right)\right)^{\tau, w} \equiv \exists w_{1} \ldots w_{n}\left[\bigwedge_{i=1}^{n} \delta\left(w_{i}\right) \wedge \bigwedge_{j=1}^{n}\left(t_{j}\right)^{\tau, w_{j}} \wedge \psi_{f}\left(w_{1}, \ldots, w_{n}, w\right)\right]
$$

where $w_{1}, \ldots, w_{n}$ are distinct variable symbols that do not occur in $\bigwedge_{j=1}^{n}\left(t_{j}\right)^{\tau, w}$.

We extend $\tau$ to a translation of all $\mathscr{L}_{1}$-formulas as follows:

6. $(\neg \phi)^{\tau} \equiv \neg \phi^{\tau}$

7. $(\phi \oslash \psi)^{\tau} \equiv \phi^{\tau} \oslash \psi^{\tau}$ for $\oslash \in\{\wedge, \vee, \rightarrow, \leftrightarrow\}$

8. $(\exists x \phi)^{\tau} \equiv \exists x\left[\delta(x) \wedge \phi^{\tau}\right]$

9. $(\forall x \phi)^{\tau} \equiv \forall x\left[\delta(x) \rightarrow \phi^{\tau}\right]$.

Let $S$ be an $\mathscr{L}_{1}$-theory and let $T$ be an $\mathscr{L}_{2}$-theory. We say that $S$ is (relatively) interpretable in $T$ if there exists a relative translation $\tau$ such that

$-T \vdash \exists x \delta(x)$

- For each function symbol $f$ of $\mathscr{L}_{1}$

$$
T \vdash \bigwedge_{i=1}^{n} \delta\left(x_{i}\right) \rightarrow \exists ! y\left[\delta(y) \wedge \psi_{f}\left(x_{1}, \ldots, x_{n}, y\right)\right] .
$$

- For each constant symbol $c$ of $\mathscr{L}_{1}$

$$
T \vdash \exists ! y\left[\delta(y) \wedge \psi_{c}(y)\right]
$$

$-T$ proves $\phi^{\tau}$ for each non-logical axiom $\phi$ of $S$. If equality is not translated as equality, then $T$ must prove the translation of each equality axiom. 
If $S$ is relatively interpretable in $T$ and $T$ is relatively interpretable in $S$, we say that $S$ and $T$ are mutually interpretable.

The following proposition summarizes important properties of relative interpretability (see Tarski et al. [18] for the details).

Proposition 1 Let $S, T$ and $U$ be computably enumerable first-order theories.

1. If $S$ is interpretable in $T$ and $T$ is consistent, then $S$ is consistent.

2. If $S$ is interpretable in $T$ and $T$ is interpretable in $U$, then $S$ is interpretable in $U$.

3. If $S$ is interpretable in $T$ and $S$ is essentially undecidable, then $T$ is essentially undecidable.

\section{Intermediate theories}

We show in our Master's thesis [12] that WBT and WTC ${ }^{-\varepsilon}$ are equivalent, i.e., they prove the same formulas. We do not include a proof of this result, but the interested reader may find the fairly straightforward proof in [12]. We also show in [12] that $\mathrm{BTQ}$ and $\mathrm{TC}^{-\varepsilon}$ are mutually interpretable. Although this result is not trivial, we omit a proof since there is another way of seeing that BTQ is mutually interpretable with Q. The theory BTQ is a fragment of the theory F, introduced first by Alfred Tarski at the end of Chapter 3 of [18]. The theory BTQ also resembles the theory $Q^{\text {bin }}$ in Visser [19]. One can view $Q^{\text {bin }}$ as the analogue of BTQ where we have an identity element and associativity has been weakened to $\forall x y[(x y) 0=x(y 0) \wedge(x y) 1=x(y 1)]$. The theory $\mathrm{F}$ differs from $\mathrm{BTQ}$ in that the axiom $\mathrm{BTQ}_{2}$ is replaced with the axioms

$$
\mathrm{F}_{2} \equiv \forall x y z[z x=z y \rightarrow x=y] \text { and } \mathrm{F}_{3} \equiv \forall x y z[x z=y z \rightarrow x=y]
$$

In Ganea [3], it is shown that $F-F_{2}$ is mutually interpretable with Q. Clearly, BTQ is interpretable in $F-F_{2}$. The other way, we can interpret $F-F_{2}$ in BTQ by simply relativizing quantification to the domain

$$
J=\{z: \forall x y[x z=y z \rightarrow x=y]\} .
$$

It follows from $\mathrm{BTQ}_{2}$ that $0,1 \in J$. We now show that $J$ is closed under $\circ$. Suppose $z, w \in J$. We need to show that $z w \in J$. We have

$$
\begin{aligned}
x(z w)=y(z w) & \Rightarrow(x z) w=(y z) w & & \left(\text { by } \mathrm{BTQ}_{1}\right) \\
& \Rightarrow x z=y z & & (\text { since } w \in J) \\
& \Rightarrow x=y & & \text { (since } z \in J) .
\end{aligned}
$$

Hence, $z w \in J$. Thus, $J$ satisfies the domain condition. Clearly, the axioms of $\mathrm{F}-\mathrm{F}_{2}$ hold on $J$. We show that the axiom $\mathrm{BTQ}_{4}$ holds on $J$. Suppose $x \in J$. By BTQ 4 , if $x \neq 0 \wedge x \neq 1$, then there exists $y$ such that $x=y 0 \vee x=y 1$. We have 


$$
\begin{aligned}
& u y=v y \Rightarrow(u y) 0=(v y) 0 \wedge(u y) 1=(v y) 1 \\
& \Rightarrow u(y 0)=v(y 0) \wedge u(y 1)=v(y 1) \quad\left(\text { by } \mathrm{BTQ}_{1}\right) \\
& \Rightarrow u x=v x \\
& \Rightarrow u=v \\
& \text { (since } x \in J \text { ). }
\end{aligned}
$$

Hence, $x \in J$ implies $y \in J$. Thus, $\mathrm{BTQ}_{4}$ holds on $J$. It is not difficult to verify that the other axioms also hold on $J$. We can thus state the following theorem which will be used implicitly to show that $D$ and $Q$ are mutually interpretable.

Theorem 1 BTQ and $Q$ are mutually interpretable.

The theories BT and $\mathrm{C}$ are examples of theories that lie strictly between $\mathrm{R}$ and $\mathrm{Q}$ w.r.t. relative interpretability. It follows from $\forall x y[x \neq y \rightarrow(x 0 \neq y 0 \wedge x 1 \neq y 1)]$ and $\forall x y[x 0 \neq y 1]$ that any model of BT or $C$ is infinite. If BT (C) were interpretable in WBT (WD), it would be interpretable in a finite sub-theory. Since any finite sub-theory of WBT or WD clearly has a finite model, we cannot interpret BT (C) in WBT (WD). We now show that BTQ is not interpretable in BT. Similar reasoning shows that D is not interpretable in $\mathrm{C}$. We let $\mathrm{BT}_{4}$ denote $\mathrm{WBT}_{3}$ and for $1 \leq i \leq 3$ we let $\mathrm{BT}_{i}$ denote $\mathrm{BTQ}_{i}$.

\section{Theorem 2 BTQ is not interpretable in BT.}

Proof Suppose $\tau$ is an interpretation of BTQ in BT. Then, there is a finite subset $\Sigma$ of the axioms of BT such that

$$
\Sigma \vdash \bigwedge_{j=1}^{4}\left(\mathrm{BTQ}_{j}\right)^{\tau} .
$$

To see that $\tau$ cannot exist, it suffices to show that the theory given by $\Sigma$ is interpretable in BT-BT 4 , which is not essentially undecidable by minimality of BTQ (see Lemma 11).

To see that $\Sigma$ is interpretable in $\mathrm{BT}-\mathrm{BT}_{4}$, it suffices to show that for each natural number $n \geq 1$, the theory $\mathrm{BT}_{\leq n}$ is interpretable in $\mathrm{BT}$. The theory $\mathrm{BT}_{\leq n}$ is like $\mathrm{BT}$ except that the axiom schema

$$
\mathrm{BT}_{4} \equiv \forall x\left[x \sqsubseteq_{\mathrm{s}} \bar{\alpha} \rightarrow \bigvee_{\gamma \in\langle\ldots \alpha\rangle} x=\bar{\gamma}\right]
$$

is limited to those $\alpha$ such that the length of $\alpha$, denoted $|\alpha|$, is bounded by $n$. To do this, we define by recursion a sequence of domains

$$
I_{1} \supseteq I_{2} \supseteq I_{3} \supseteq \ldots \ldots
$$

such that we obtain an interpretation of $\mathrm{BT}_{\leq n}$ in $\mathrm{BT}$ by simply relativizing quantification to $I_{n}$. So, we proceed to construct these domains. We will omit parentheses most of the time since we have the axiom

$$
\mathrm{BT}_{1} \equiv \forall x y z[(x y) z=x(y z)]
$$


in $\mathrm{BT}-\mathrm{BT}_{4}$.

Construction of $I_{1}$ :

We let

$$
A_{1}=\{y: \forall x[x y 0 \neq 0 \wedge x y 1 \neq 1]\} .
$$

Suppose $x 00=0 \vee x 01=1$. Then, $11 x 00=110 \vee 11 x 01=111 . \mathrm{By} \mathrm{BT}_{2}$, we then have $11 x 0=11$, contradicting $\mathrm{BT}_{3}$. Hence, $0 \in A_{1}$. By similar reasoning, we have $1 \in A_{1}$. We now show that $A_{1}$ is closed under o. Suppose $y, y^{\prime} \in A_{1}$. By $\mathrm{BT}_{1}$, we have

$$
x\left(y y^{\prime}\right) 0=(x y) y^{\prime} 0 \text { and } x\left(y y^{\prime}\right) 1=(x y) y^{\prime} 1 .
$$

Hence, $x\left(y y^{\prime}\right) 0=0 \vee x\left(y y^{\prime}\right) 1=1$ contradicts $y^{\prime} \in A_{1}$. Thus, $y y^{\prime} \in A_{1}$. Hence, $A_{1}$ is closed under $\circ$.

We let

$$
B_{1}=\left\{x \in A_{1}: x 0 \neq 0 \wedge x 1 \neq 1\right\}
$$

Suppose $00=0 \vee 01=1$. Then, $1100=110 \vee 1101=111$. By BT 2 , we then have $110=11$, contradicting $\mathrm{BT}_{3}$. Thus, $0 \in B_{1}$. By similar reasoning, we have $1 \in B_{1}$. We now show that $B_{1}$ is closed under o. Suppose $y, y^{\prime} \in B_{1}$. We observe that

$$
y y^{\prime} 0=0 \vee y y^{\prime} 1=1
$$

contradicts $y^{\prime} \in A_{1}$. Hence, $y y^{\prime} \in B_{1}$. Thus, $B_{1}$ is closed under $\circ$.

We let

$$
I_{1}=\left\{y \in B_{1}: \forall x \in B_{1}[x y \neq 0 \wedge x y \neq 1]\right\} .
$$

We clearly cannot have $x 1=0 \vee x 0=1$ since $1 x 1=10 \vee 1 x 0=11$ would follow, contradicting $\mathrm{BT}_{3}$. It follows from the definition of $B_{1}$ that we have

$$
\forall x \in B_{1}[x 0 \neq 0 \wedge x 1 \neq 1]
$$

Hence, $0,1 \in I_{1}$. We now show that $I_{1}$ is closed under $\circ$. Suppose $y, y^{\prime} \in I_{1}, x \in B_{1}$ and $x\left(y y^{\prime}\right)=0 \vee x\left(y y^{\prime}\right)=1$. Since $y \in I_{1} \subseteq B_{1}$ and $B_{1}$ is closed under $\circ$, we have $x y \in B_{1}$. By $\mathrm{BT}_{1}$, we have have $(x y) y^{\prime}=0 \vee(x y) y^{\prime}=1$, contradicting $y^{\prime} \in I_{1}$. Hence, $y y^{\prime} \in I_{1}$. Thus, $I_{1}$ is closed under o. This means that $I_{1}$ satisfies the domain conditions.

We interpret $B T_{\leq 1}$ in $B T-B T_{4}$ by simply relativizing quantification to $I_{1}$. Since $B T_{1}$, $\mathrm{BT}_{2}$ and $\mathrm{BT}_{3}$ are universal sentences that are theorems of $\mathrm{BT}-\mathrm{BT}_{4}$, they hold in $I_{1}$. It remains to show that $B T-B T_{4}$ proves the translation of

(1) $\forall x[x \sqsubseteq s 0 \rightarrow x=0]$

(2) $\forall x[x \sqsubseteq \mathrm{s} 1 \rightarrow x=1]$. 
We only show (1). The case (2) is handled similarly. So, suppose $x \in I_{1}$ and the translation of $x \sqsubseteq$ s 0 holds. We then have one of the following cases for some $z, w \in I_{1}$ :

(i) $0=x$

(ii) $0=z x$

(iii) $0=x w$

(iv) $0=(z x) w$

(v) $0=z(x w)$.

We notice that (ii)-(v) contradict the definition of $I_{1}$. We thus see that the translation of (1) is a theorem of BT-BT . Similarly, the translation of (2) is a theorem of BT-BT 4 . Construction of $I_{n+1}$ :

Let $\Gamma_{n}$ denote the set of all nonempty biterals of length at most $n$. Let $\sqsubseteq_{s}^{I_{n}}$ denote the realization of $\sqsubseteq_{\mathrm{s}}$ in $I_{n}$, i.e.

$$
x \sqsubseteq_{\mathrm{s}}^{I_{n}} y \equiv y=x \vee \exists u v \in I_{n}[y=u x \vee y=x v \vee y=u x v] .
$$

We assume $I_{n}$ has been constructed and satisfies

$$
\bigwedge_{\alpha \in \Gamma_{n}} \forall x \in I_{n}\left[x \sqsubseteq_{\mathrm{s}}^{I_{n}} \bar{\alpha} \rightarrow \bigvee_{\gamma \in\langle\ldots \alpha\rangle} x=\bar{\gamma}\right] .
$$

We let

$$
\begin{aligned}
I_{n+1}= & \left\{y \in I_{n}: \bigwedge_{\alpha \in \Gamma_{n}}\left(\forall x \in I_{n}\left[x y=\bar{\alpha} 0 \rightarrow \bigvee_{\gamma, \delta \in\langle\ldots \alpha \mathbf{0}\rangle}(x=\bar{\gamma} \wedge y=\bar{\delta})\right]\right.\right. \\
& \left.\left.\wedge \forall x \in I_{n}\left[x y=\bar{\alpha} 1 \rightarrow \bigvee_{\gamma, \delta \in\langle\ldots \alpha \mathbf{1}\rangle}(x=\bar{\gamma} \wedge y=\bar{\delta})\right]\right)\right\} .
\end{aligned}
$$

By $\mathrm{BT}_{3}$, we cannot have $x 1=\bar{\alpha} 0 \vee x 0=\bar{\alpha} 1$. By $\mathrm{BT}_{2}$, we have

$$
x 0=\bar{\alpha} 0 \Rightarrow x=\bar{\alpha}
$$

and

$$
x 1=\bar{\alpha} 1 \Rightarrow x=\bar{\alpha}
$$

Hence, $0,1 \in I_{n+1}$. We now show that $I_{n+1}$ is closed under $\circ$. Suppose $y_{0}, y_{1} \in I_{n+1}$. Let $x \in I_{n}$. We observe that $x y_{0} \in I_{n}$ since $x, y_{0} \in I_{n}$ and $I_{n}$ is closed under $\circ$. By $\mathrm{BT}_{1}$, we have $x\left(y_{0} y_{1}\right)=\left(x y_{0}\right) y_{1}$. Then

$$
\begin{aligned}
x\left(y_{0} y_{1}\right)=\bar{\alpha} 0 & \Rightarrow \bigvee_{\gamma, \delta \in\{\ldots \alpha \mathbf{0}\rangle}\left(x y_{0}=\bar{\gamma} \wedge y_{1}=\bar{\delta}\right) & & \left(y_{1} \in I_{n+1} \text { and } x y_{0} \in I_{n}\right) \\
& \Rightarrow \bigvee_{\zeta, \eta, \delta \in\{\ldots \alpha \mathbf{0}\rangle}\left(x=\bar{\zeta} \wedge y_{0}=\bar{\eta} \wedge y_{1}=\bar{\delta}\right) & & \left(x, y_{0} \in I_{n} \text { and } \gamma \in \Gamma_{n}\right)
\end{aligned}
$$

and

$$
\begin{aligned}
x\left(y_{0} y_{1}\right)=\bar{\alpha} 1 & \Rightarrow \bigvee_{\gamma, \delta \in\{\ldots \alpha \mathbf{1}\rangle}\left(x y_{0}=\bar{\gamma} \wedge y_{1}=\bar{\delta}\right) & \left(y_{1} \in I_{n+1} \text { and } x y_{0} \in I_{n}\right) \\
& \Rightarrow \bigvee_{\zeta, \eta, \delta \in\{\ldots \alpha \mathbf{1}\rangle}\left(x=\bar{\zeta} \wedge y_{0}=\bar{\eta} \wedge y_{1}=\bar{\delta}\right) & \left(x, y_{0} \in I_{n} \text { and } \gamma \in \Gamma_{n}\right) .
\end{aligned}
$$


It is not difficult to see that $\mathrm{BT}-\mathrm{BT}_{4}$ proves each instance of the axiom schemas

$\mathrm{WBT}_{1} \bar{\alpha} \bar{\beta}=\overline{\alpha \beta}$

$\mathrm{WBT}_{2} \bar{\alpha} \neq \bar{\beta}$ if $\alpha \neq \beta$.

This implies

$$
x\left(y_{0} y_{1}\right)=\bar{\alpha} 0 \Rightarrow \bigvee_{\beta, \zeta \in\langle\ldots \alpha \mathbf{0}\rangle}\left(x=\bar{\zeta} \wedge y_{0} y_{1}=\bar{\beta}\right)
$$

and

$$
x\left(y_{0} y_{1}\right)=\bar{\alpha} 1 \Rightarrow \bigvee_{\beta, \zeta \in\langle\ldots \alpha \mathbf{1}\rangle}\left(x=\bar{\zeta} \wedge y_{0} y_{1}=\bar{\beta}\right)
$$

Hence, $y_{0} y_{1} \in I_{n+1}$. Thus, $I_{n+1}$ is closed under $\circ$. This proves that $I_{n+1}$ satisfies the domain conditions. It is clear that we get an interpretation of $\mathrm{BT}_{\leq n+1}$ in $\mathrm{BT}-\mathrm{BT}_{4}$ by simply relativizing quantification to $I_{n+1}$.

By a similar argument, we see that D is not interpretable in $C$.

Theorem 3 D is not interpretable in $C$.

The theories C and BT are strictly between R and Q w.r.t. interpretability. A natural question is whether they are mutually interpretable. It is not difficult to see that our interpretation of WD in WBT given at the end of Sect. 4 is also an interpretation of C in BT. It is not clear however whether BT is interpretable in C.

Open Problem 1 Is BT interpretable in C?

\section{The theory WD}

We now show that the theory WD is mutually interpretable with $R$. We give an interpretation of $R$ in WD. To show that WD is interpretable in $R$ we invoke the result of Visser [20]: a recursively enumerable theory is interpretable in $R$ if and only if it is locally finitely satisfiable (each finite sub-theory has a finite model). We also show how to interpret WD in WBT, from which follows that WD is interpretable in R since WBT and $\mathrm{WTC}^{-\varepsilon}$ are equivalent, and $\mathrm{WTC}^{-\varepsilon}$ and $\mathrm{R}$ are mutually interpretable (see Fig. 2). The theory WD is purely universally axiomatised, in contrast to WTC ${ }^{-\varepsilon}$ which has $\Pi_{2}$-axioms.

Definition 1 The first-order theory WD contains the following non-logical axioms: for each $\alpha, \beta \in\{\mathbf{0}, \mathbf{1}\}^{+}$

$$
\begin{aligned}
& \mathrm{WD}_{1} \bar{\alpha} \bar{\beta}=\overline{\alpha \beta} \\
& \mathrm{WD}_{2} \bar{\alpha} \neq \bar{\beta} \quad \text { if } \alpha \neq \beta \\
& \mathrm{WD}_{3} \forall x\left[x \preceq \bar{\alpha} \leftrightarrow \bigvee_{\gamma \leq^{\mathfrak{D} \alpha}} x=\bar{\gamma}\right]
\end{aligned}
$$


We start by showing that a theory of arithmetic we call $\mathrm{R}^{-}$is interpretable in WD. The theory $\mathrm{R}^{-}$has been shown to be mutually interpretable with $\mathrm{R}$ (see Jones and Shepherdson [8]).

Definition 2 The first-order theory $\mathrm{R}^{-}$contains the following non-logical axioms: for each $n, m \in \mathbb{N}$

$$
\begin{aligned}
& \mathrm{R}^{-1} \bar{n} \times \bar{m}=\overline{n m} \\
& \mathrm{R}^{-2} \bar{n} \neq \bar{m} \quad \text { if } n \neq m \\
& \mathrm{R}^{-3} \quad \forall x\left[x \leq \bar{n} \leftrightarrow \bigvee_{k \leq n} x=\bar{k}\right] .
\end{aligned}
$$

The most difficult step in interpreting $\mathrm{R}^{-}$in WD is translating $\times$. We start by proving two lemmas which show how we intend to translate $\times$. In the structure $\mathfrak{D}$, we can associate each natural number $k$ with the bit string $\mathbf{1}^{k} \mathbf{0}$. For $n \neq 0$ and $m>1$, we can then translate

$$
\bar{n} \times \bar{m}=\overline{n m}
$$

by saying that the sequence

$$
\left(\overline{1^{n}}, \overline{1^{n+n}}, \overline{1^{n+n+n}}, \ldots, \overline{1^{n m}}\right)
$$

exists. The formulas given in Lemmas 1 and 2 try to capture this way of viewing multiplication. But we have to be careful since we are reasoning in a weak theory. For example, we lack full associativity. For readability, we dispense with parentheses whenever possible and expect terms to be read from right to left. That is, $x y z$ should be regarded as shorthand for $(x y) z$. Although we do not have full associativity, by $\mathrm{WD}_{1}$, we have WD $\vdash(\bar{\alpha} \bar{\beta}) \bar{\gamma}=\bar{\alpha}(\bar{\beta} \bar{\gamma})$ for all $\alpha, \beta, \gamma \in\{\mathbf{0}, \mathbf{1}\}^{+}$. Then, by $\mathrm{WD}_{3}$, we have

$$
\mathrm{WD} \vdash \forall x \preceq \bar{\alpha} \forall y \preceq \bar{\beta} \forall z \preceq \bar{\gamma}[(x y) z=x(y z)] .
$$

The bounded quantifiers in Lemmas 1 and 2 are there to make full use of the axiom scheme $\mathrm{WD}_{3}$.

Lemma 1 Let $n \neq 0$ and $m \geq 2$. Then, WD proves

(1) $\overline{1^{n m}} \preceq \overline{1^{n m}}$ and $\overline{1^{n}}, \overline{1^{m}}$ and $\overline{1^{n m}}$ are the unique elements such that

(a) $\overline{1^{n}} \preceq \overline{1^{n} 0} \wedge \overline{1^{n} 0}=\overline{1^{n}} 0$

(b) $\overline{1^{m}} \preceq \overline{1^{m} 0} \wedge \overline{1^{m} 0}=\overline{1^{m}} 0$

(c) $\overline{1^{n m}} \preceq \overline{1^{n m} 0} \wedge \overline{1^{n m} 0}=\overline{1^{n m}} 0$.

Furthermore, let

$$
\mathfrak{w} \equiv 000 \overline{1^{n}} 0100 \overline{1^{n+n}} 01100 \overline{1^{n+n+n}} 011100 \ldots 00 \overline{1^{n m}} 0 \overline{1^{m}} 00
$$

Then, $\mathfrak{w}$ provably (in WD) satisfies: 
(2) $\exists u \preceq \mathfrak{w}\left[u 00 \overline{1^{n m}} 0 \overline{1^{m}} 00 \preceq \mathfrak{w}\right]$

(3) it holds that $\left(\forall w^{\prime} \preceq \mathfrak{w}\right)\left(\forall y^{\prime} \preceq \overline{1^{m}}\right)\left(\forall z^{\prime} \preceq \overline{1^{n m}}\right) \Psi_{0}\left(w^{\prime}, y^{\prime}, z^{\prime}\right)$ where

$$
\begin{aligned}
\Psi_{0}\left(w^{\prime}, y^{\prime}, z^{\prime}\right) \equiv & \left(y^{\prime} \neq 1 \wedge w^{\prime} 00 z^{\prime} 0 y^{\prime} 00 \preceq \mathfrak{w}\right) \rightarrow \\
& \left(\exists w^{\prime \prime} \preceq \mathfrak{w}\right)\left(\exists y^{\prime \prime} \preceq y^{\prime}\right)\left(\exists z^{\prime \prime} \preceq \overline{1^{n m}}\right)[ \\
z^{\prime}= & \left.z^{\prime \prime} \overline{1^{n}} \wedge y^{\prime}=y^{\prime \prime} 1 \wedge w^{\prime \prime} 00 z^{\prime \prime} 0 y^{\prime \prime} 00 \preceq \mathfrak{w} \wedge \Phi_{0}\left(y^{\prime \prime}, z^{\prime \prime}\right)\right]
\end{aligned}
$$

and

$$
\Phi_{0}\left(y^{\prime \prime}, z^{\prime \prime}\right) \equiv y^{\prime \prime}=1 \rightarrow z^{\prime \prime}=\overline{1^{n}}
$$

Proof Since we have the axiom schema $W D_{1}$, we can skip parentheses in $\mathfrak{w}$. Clause (1) holds due to the axiom schemas $W D_{3}$ and $W D_{2}$. We verify clause (a). By the rightleft implication of $\mathrm{WD}_{3}$, we have $\overline{1^{n}} \preceq \overline{1^{n} 0}$. By how biterals are defined, we have $\overline{1^{n} 0}=\overline{1^{n}} 0$. For uniqueness, suppose

$$
x \preceq \overline{1^{n} 0} \wedge x 0=\overline{1^{n} 0} .
$$

By $W_{3}$, we have

$$
x \preceq \overline{1^{n} 0} \rightarrow \bigvee_{\gamma \preceq^{\mathfrak{D}} 1^{n} 0} x=\bar{\gamma}
$$

Then, by $\mathrm{WD}_{2}$, in order for $x 0=\overline{1^{n} 0}$ to hold we must have $x=\overline{1^{n}}$. By similar reasoning, one verifies clauses (b) and (c).

Clause (2) holds due to the axiom schema $W_{1}$ and the right-left implication of $\mathrm{WD}_{3}$. Let

$$
u=000 \overline{1^{n}} 0100 \overline{1^{n+n}} 01100 \overline{1^{n+n+n}} 011100 \ldots 00 \overline{1^{n(m-1)}} 0 \overline{1^{m-1}} .
$$

By the right-left implication of $\mathrm{WD}_{3}$, we have $u \preceq \mathfrak{w}$. By $\mathrm{WD}_{1}$, we have

$$
u 00 \overline{1^{n m}} 0 \overline{1^{m}} 00=\mathfrak{w}
$$

Then, by the right-left implication of $\mathrm{WD}_{3}$, we have

$$
u 00 \overline{1^{n m}} 0 \overline{1^{m}} 00 \preceq \mathfrak{w} .
$$

To see that clause (3) holds, suppose

$$
w^{\prime} \preceq \mathfrak{w} \wedge z^{\prime} \preceq \overline{1^{n m}} \wedge y^{\prime} \preceq \overline{1^{m}} \wedge y^{\prime} \neq 1 \wedge w^{\prime} 00 z^{\prime} 0 y^{\prime} 00 \preceq \mathfrak{w}
$$

holds. By the axiom schemas $\mathrm{WD}_{3}$ and $\mathrm{WD}_{2}$, the third and fourth conjunct of $\left(^{*}\right)$ imply $y^{\prime}=\overline{1^{k+1}}$ where $2 \leq k+1 \leq m$. Given $w^{\prime} \preceq \mathfrak{w} \wedge z^{\prime} \preceq \overline{1^{n m}}$, the axiom schema WD 3 gives us a set $\Gamma$ of pairs $(a, \bar{b})$ where $a \preceq \mathfrak{w}$ and $b \preceq \overline{1^{n m}}$ such that $\left(w^{\prime}, z^{\prime}\right) \in \Gamma$. For 
each pair $(a, b) \in \Gamma$, we use $\mathrm{WD}_{1}$ to compute $a 00 b 0 y^{\prime} 00$ and then use the left-right implication of $\mathrm{WD}_{3}$ and $\mathrm{WD}_{2}$ to determine whether $a 00 b 0 y^{\prime} 00 \preceq \mathfrak{w}$. We are then led to the conclusion

- $w^{\prime}=000 \overline{1^{n}} 0100 \overline{1^{n+n}} 01100 \overline{1^{n+n+n}} 011100 \ldots 00 \overline{1^{n k}} 0 \overline{1^{k}}$

$-z^{\prime}=\overline{1^{n(k+1)}}$.

By $W_{1}$ and the right-left implication of $W_{3}$, we have

(I) $w^{\prime}=000 \overline{1^{n}} 0100 \overline{1^{n+n}} 01100 \overline{1^{n+n+n}} 011100 \ldots 00 \overline{1^{n k}} 0 \overline{1^{k}} \preceq \mathfrak{w}$

(II) $z^{\prime}=\overline{1^{n(k+1)}}=\overline{1^{n k}} \overline{1^{n}}$

(III) $y^{\prime}=\overline{1^{k+1}}=\overline{1^{k}} 1$.

We can then let

$$
w^{\prime \prime}=000 \overline{1^{n}} 0100 \overline{1^{n+n}} 01100 \overline{1^{n+n+n}} 011100 \ldots 00 \overline{1^{n(k-1)}} 0 \overline{1^{k-1}}
$$

and $z^{\prime \prime}=\overline{1^{n k}} \wedge y^{\prime \prime}=\overline{1^{k}}$. If $k=1$, we let $w^{\prime \prime}=0$. If $\overline{1^{k}}=1$, then by $\mathrm{WD}_{2}$ we must have $k=1$. We thus see that clause (3) holds.

\section{Lemma 2 Let}

$$
\begin{aligned}
& \phi_{M}(X, Y, Z) \equiv((X=0 \vee Y=0) \wedge Z=0) \vee(Y=10 \wedge Z=X) \\
& \vee(X \neq 0 \wedge Y \neq 0 \wedge Y \neq 10 \wedge \exists x \preceq X \exists y \preceq Y \exists z \preceq Z \exists w \\
& \quad[X=x 0 \wedge Y=y 0 \wedge Z=z 0 \wedge z \preceq z \\
& \wedge \exists u \preceq w[u 00 z 0 y 00 \preceq w] \\
& \left.\left.\wedge\left(\forall w^{\prime} \preceq w\right)\left(\forall y^{\prime} \preceq y\right)\left(\forall z^{\prime} \preceq z\right) \Psi\left(w^{\prime}, y^{\prime}, z^{\prime}, x, w\right)\right]\right)
\end{aligned}
$$

where

$$
\begin{aligned}
& \Psi\left(w^{\prime}, y^{\prime}, z^{\prime}, x, w\right) \equiv\left(y^{\prime} \neq 1 \wedge w^{\prime} 00 z^{\prime} 0 y^{\prime} 00 \preceq w\right) \\
& \rightarrow\left(\exists w^{\prime \prime} \preceq w\right)\left(\exists y^{\prime \prime} \preceq y^{\prime}\right)\left(\exists z^{\prime \prime} \preceq z\right) \\
& {\left[z^{\prime}=z^{\prime \prime} x \wedge y^{\prime}=y^{\prime \prime} 1 \wedge w^{\prime \prime} 00 z^{\prime \prime} 0 y^{\prime \prime} 00 \preceq w \wedge \Phi\left(y^{\prime \prime}, z^{\prime \prime}, x\right)\right]}
\end{aligned}
$$

and

$$
\Phi\left(y^{\prime \prime}, z^{\prime \prime}, x\right) \equiv y^{\prime \prime}=1 \rightarrow z^{\prime \prime}=x
$$

Then, for each natural number $n$ and $m$, we have

$$
W D \vdash \forall Z\left[\phi_{M}\left(\overline{1^{n} 0}, \overline{1^{m} 0}, Z\right) \leftrightarrow Z=\overline{1^{n m} 0}\right]
$$

Proof We start by showing that

$$
\mathrm{WD} \vdash \forall Z\left[Z=\overline{1^{n m} 0} \rightarrow \phi_{M}\left(\overline{1^{n} 0}, \overline{1^{m} 0}, Z\right)\right] .
$$


- If $n=0$ or $m=0$, then the first disjunct in the definition of $\phi_{M}(X, Y, Z)$ holds.

- If $m=1$, then the second disjunct in the definition of $\phi_{M}(X, Y, Z)$ holds.

- If $n \neq 0$ and $m \geq 2$, then by $\mathrm{WD}_{1}$ and $\mathrm{WD}_{2}$ we have

$$
\overline{1^{n}} 0=\overline{1^{n} 0} \neq 0 \wedge \overline{1^{m}} 0=\overline{1^{m} 0} \neq 0 \wedge \overline{1^{m} 0} \neq 10 .
$$

Then, by Lemma 1, the third disjunct in the definition of $\phi_{M}(X, Y, Z)$ holds since we can choose

$$
w=000 \overline{1^{n}} 0100 \overline{1^{n+n}} 01100 \ldots 00 \overline{1^{n m}} 0 \overline{1^{m}} 00
$$

Thus, (**) holds.

We now prove that

$$
\mathrm{WD} \vdash \forall Z\left[\phi_{M}\left(\overline{1^{n} 0}, \overline{1^{m} 0}, Z\right) \rightarrow Z=\overline{1^{n m} 0}\right] . \quad(* * *)
$$

So, suppose $\phi_{M}\left(\overline{1^{n} 0}, \overline{1^{m} 0}, Z\right)$ holds. If $n=0$ or $m \leq 1$, then it follows from the definition of $\phi_{M}\left(\overline{1^{n} 0}, \overline{1^{m} 0}, Z\right)$ that $Z=\overline{1^{n m} 0}$. We therefore assume that $n \neq 0$ and $m>1$. Then, there exist $x \preceq \overline{1^{n} 0}, y_{1} \preceq \overline{1^{m} 0}$ and $z_{1} \preceq Z$ such that

$$
\overline{1^{n} 0}=x 0 \wedge \overline{1^{m} 0}=y_{1} 0 \wedge Z=z_{1} 0 \wedge z_{1} \preceq z_{1} .
$$

By $W D_{3}$ and $W D_{2}$, we have

$$
x=\overline{1^{n}} \wedge y_{1}=\overline{\mathbf{1}^{m}} .
$$

Furthermore, by the definition of $\phi_{M}\left(\overline{1^{n} 0}, \overline{1^{m} 0}, Z\right)$, there exist $w$ and $w_{1} \preceq w$ such that

$$
w_{1} 00 z_{1} 0 y_{1} 00 \preceq w .
$$

By $W_{1}$, it suffices to show that $z_{1}=\overline{1^{n m}}$ to conclude that $Z=\overline{1^{n m} 0}$. By the axiom schema $\mathrm{WD}_{2}$, we have $\overline{1^{m}} \neq 1$ since $m>1$. By the axiom schema $\mathrm{WD}_{3}$, we have $\overline{1^{m}} \preceq \overline{1^{m}}$. Then, $\Psi\left(w_{1}, y_{1}, z_{1}, x, w\right)$ in the definition of $\phi_{M}\left(\overline{1^{n} 0}, \overline{1^{m} 0}, Z\right)$ implies that there exist $w_{2} \preceq w, y_{2} \preceq y_{1}$ and $z_{2} \preceq z_{1}$ such that

$$
z_{1}=z_{2} \overline{1^{n}} \wedge y_{1}=y_{2} 1 \wedge w_{2} 00 z_{2} 0 y_{2} 00 \preceq w .
$$

By the axiom schemas $W_{3}$ and $W_{2}$, we must have

$$
y_{2}=\overline{1^{m-1}} .
$$

If $m-1=1$, then $\Phi\left(y_{2}, z_{2}, x\right)$ in the definition of $\phi_{M}\left(\overline{1^{n} 0}, \overline{1^{m} 0}, Z\right)$ implies $z_{2}=\overline{1^{n}}$. By $W D_{1}$, this implies

$$
z_{1}=z_{2} \overline{1^{n}}=\overline{1^{n}} \overline{1^{n}}=\overline{1^{n+n}}=\overline{1^{n m}}
$$


since $m=2$. If $m-1 \neq 1$, we use $\Psi\left(w_{2}, y_{2}, z_{2}, x, w\right)$ to conclude that there exist

$$
w_{3} \preceq w, y_{3} \preceq y_{2} \text { and } z_{3} \preceq z_{1}
$$

such that

$$
z_{2}=z_{3} \overline{1^{n}} \wedge y_{2}=y_{3} 1 \wedge w_{3} 00 z_{3} 0 y_{3} 00 \preceq w
$$

By the axiom schemas $W_{3}$ and $W_{2}$, we must have

$$
y_{3}=\overline{1^{m-2}} \text {. }
$$

If $m-2=1$, then by $\Phi\left(y_{3}, z_{3}, x\right)$ we have $z_{3}=\overline{1^{n}}$. By $\mathrm{WD}_{1}$, this implies

$$
z_{1}=z_{2} \overline{1^{n}}=\left(z_{3} \overline{1^{n}}\right) \overline{1^{n}}=\overline{1^{n}} \overline{1^{n}} \overline{1^{n}}=\overline{1^{n+n+n}}=\overline{1^{n m}}
$$

since $m=3$. If $m-2 \neq 1$, we use $\Psi\left(w_{3}, y_{3}, z_{3}, x, w\right)$ to repeat this procedure. We notice that after a finite number of steps, we have that there exist $w_{m} \preceq w, y_{m} \preceq y_{m-1}$ and $z_{m} \preceq z_{1}$ such that

$$
z_{m-1}=z_{m} \overline{1^{n}} \wedge y_{m-1}=\overline{1^{m-(m-2)}} \wedge y_{m-1}=y_{m} 1 \wedge w_{m} 00 z_{m} 0 y_{m} 00 \preceq w .
$$

By the axiom schemas $W_{3}$ and $W_{2}$, we must have

$$
y_{m}=1 \text {. }
$$

The condition $\Phi\left(y_{m}, z_{m}, x\right)$ in $\Psi\left(w_{m-1}, y_{m-1}, z_{m-1}, x, w\right)$ then implies

$$
z_{m}=\overline{1^{n}}
$$

By backtracking, we observe that

$$
z_{1}=\overline{1^{n}} \overline{1^{n}} \ldots \overline{1^{n}}
$$

where $\overline{1^{n}}$ occurs $m$ times. It then follows from the axiom schema $W_{1}$ that $z_{1}=\overline{1^{n m}}$. Thus, by $W_{1}$, we have $Z=\overline{1^{n m} 0}$. Hence, (***) holds.

Theorem $4 R^{-}$is interpretable in WD.

Proof We choose the domain $\delta(x) \equiv x=x$. We translate, $0, \mathrm{~S}, \times$ and $\leq$ as follows:

$$
\begin{aligned}
\phi_{0}(x) \equiv & x=0 \\
\phi_{\mathrm{S}}(x, y) \equiv & y=1 x \\
\phi_{\times}(x, y, z) \equiv & \left(\exists ! w\left[\phi_{M}(x, y, w)\right] \wedge \phi_{M}(x, y, z)\right) \vee \\
& \left(\neg \exists ! w\left[\phi_{M}(x, y, w)\right] \wedge z=0\right)
\end{aligned}
$$




$$
\phi_{\leq}(x, y) \equiv x=0 \vee \exists z \exists w \preceq y[x=z 0 \wedge y=w 0 \wedge z \preceq w] .
$$

The formula $\phi_{M}$ is defined in Lemma 2. By the axiom schema $\mathrm{WD}_{1}$, our translation of S implies that we translate each numeral $\bar{n}$ as the biteral $\overline{1^{n} 0}$. By the axiom schema $W D_{2}$, the translation of each instance of the axiom schema $R^{-2}$ is a theorem of WD.

We now show that the translation of each instance of the axiom schema $R^{-1}$ is a theorem of WD. By Lemma 2, for each natural number $n$ and $m$, we have

$$
\mathrm{WD} \vdash \exists ! w\left[\phi_{M}\left(\overline{1^{n} 0}, \overline{1^{m} 0}, w\right] \wedge \phi_{M}\left(\overline{1^{n} 0}, \overline{1^{m} 0}, \overline{1^{n m} 0}\right) .\right.
$$

Hence

$$
\mathrm{WD} \vdash \phi_{\times}\left(\overline{1^{n} 0}, \overline{1^{m} 0}, \overline{1^{n m} 0}\right)
$$

Thus, the translation of each instance of the axiom schema $R^{-1}$ is a theorem of WD. We observe that WD can have models where not all elements in the universe are realization of terms of the form $\overline{1^{k} 0}$. Hence, the condition

$$
\neg \exists ! w\left[\phi_{M}(x, y, w)\right] \wedge z=0
$$

in the definition of $\phi_{\times}(x, y, z)$ ensures that $\times$ is translated as a total function.

We now show that the translation of each instance of the axiom schema $\mathrm{R}^{-3}$ is a theorem of WD. We have

$$
\begin{aligned}
\phi_{\leq}\left(x, \overline{1^{n} 0}\right) & \Leftrightarrow x=0 \vee \exists z \exists w \preceq \overline{1^{n} 0}\left[x=z 0 \wedge \overline{1^{n} 0}=w 0 \wedge z \preceq w\right] \\
& \Leftrightarrow x=0 \vee \exists z\left[x=z 0 \wedge z \preceq \overline{1^{n}}\right] \\
& \Leftrightarrow x=0 \vee \bigvee_{\gamma \preceq^{\mathfrak{D}} 1^{n} x=\bar{\gamma} 0} \\
& \Leftrightarrow x=0 \vee \bigvee_{\gamma \preceq^{\mathfrak{D}} 1^{n}} x=\overline{\gamma 0} .
\end{aligned}
$$

The last equivalence is due to how biterals are defined. We thus see that the translation of each instance of the axiom schema $\mathrm{R}^{-3}$ is a theorem of WD.

Theorem 5 WD and $R$ are mutually interpretable.

Proof We have shown that $\mathrm{R}^{-}$is interpretable in WD. Since $\mathrm{R}^{-}$and $\mathrm{R}$ are mutually interpretable, $R$ is interpretable in WD. To see that WD is interpretable in $R$, we first observe that WD is locally finitely satisfiable, i.e., any finite subset of the axioms has a finite model. In [20], Albert Visser shows that a recursively enumerable theory is interpretable in R if and only if it is locally finitely satisfiable. Hence, WD is interpretable in $R$. Thus, WD and $R$ are mutually interpretable.

We could have shown that WD is interpretable in $\mathrm{R}$ by showing that it is interpretable in $\mathrm{WTC}^{-\varepsilon}$, which we know is mutually interpretable with R. Since we in our Master's thesis [12] show that WBT and $\mathrm{WTC}^{-\varepsilon}$ are equivalent, it suffices to show how to interpret WD in WBT. We choose the domain $\delta(x) \equiv x=x$. We translate 0,1 , o and $\preceq$ as follows: 


$$
\begin{aligned}
\phi_{0}(x) & \equiv x=0 \\
\phi_{1}(x) & \equiv x=1 \\
\phi_{\circ}(x, y, z) & \equiv z=x y \\
\phi_{\preceq}(x, y) & \equiv y=x \vee \exists z[y=x z] .
\end{aligned}
$$

Clearly, the translation of each instance of the axiom schemas $W_{1}$ and $W D_{2}$ is a theorem of WBT. We now show that the translation of each instance of the axiom schema $W_{3}$ is a theorem of WBT. We have

$$
\begin{aligned}
& \phi_{\preceq}(x, \bar{\alpha}) \Leftrightarrow \bar{\alpha}=x \vee \exists z[\bar{\alpha}=x z] \\
& \Leftrightarrow \bar{\alpha}=x \vee \exists z\left[x \sqsubseteq_{\mathrm{s}} \bar{\alpha} \wedge z \sqsubseteq_{\mathrm{s}} \bar{\alpha} \wedge \bar{\alpha}=x z\right] \text { (by def. of } \sqsubseteq_{\mathrm{s}} \text { ) } \\
& \Leftrightarrow \bar{\alpha}=x \vee \\
& \bigvee_{\beta, \gamma \in\langle\ldots \alpha\rangle}(x=\bar{\beta} \wedge z=\bar{\gamma} \wedge \bar{\beta} \bar{\gamma}=\bar{\alpha}) \quad\left(\mathrm{WBT}_{3}\right) \\
& \Leftrightarrow \bigvee_{\beta \preceq^{\mathcal{D}} \alpha} x=\bar{\beta} \quad\left(\mathrm{WBT}_{1}, \mathrm{WBT}_{2}\right) .
\end{aligned}
$$

We thus see that the translation of each instance of the axiom schema $\mathrm{WD}_{3}$ is a theorem of WBT. Hence, WD is interpretable in WBT.

\section{The theory D}

We now show that the theory $\mathrm{D}$ is mutually interpretable with $\mathrm{Q}$. In contrast to $\mathrm{Q}$ and $\mathrm{TC}^{-\varepsilon}$, which have $\Pi_{2}$-axioms, the theory $\mathrm{D}$ is purely universally axiomatised. When interpreting one theory into another, handling existential quantifiers can become cumbersome. This is clearly illustrated in the proof of Theorem 6. Therefore, having a theory with purely universal axiomatization and that is mutually interpretable with $\mathrm{Q}$ could be advantageous in some circumstances.

Definition 3 The first-order theory D is defined by the following non-logical axioms:

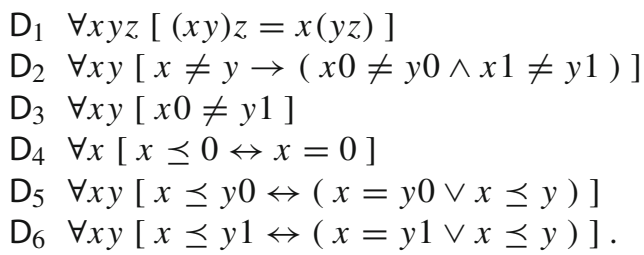

For $\mathrm{D}$ to be an extension of WD, we need the axiom $\forall x[x \preceq 1 \leftrightarrow x=1]$. The theory $\mathrm{D}$ extended with this axiom is what we call $\mathrm{D}$ at page3. The next lemma shows why we have decided to not include this axiom. We could very well have replaced $\mathrm{D}_{4}$ by $\forall x[x \preceq 1 \leftrightarrow x=1]$. The proof of the next lemma also illustrates some of the advantages of not having to worry about existential quantifiers when defining a domain.

Lemma $3 D+\forall x[x \preceq 1 \leftrightarrow x=1]$ is interpretable in $D$. 
Proof We translate 0,1 , ○ and $\preceq$ as

$$
\begin{aligned}
\phi_{0}(x) & \equiv x=0100 \\
\phi_{1}(x) & \equiv x=0110 \\
\phi_{\preceq}(x, y) & \equiv x \preceq y \\
\phi_{\circ}(x, y, z) & \equiv x y=z .
\end{aligned}
$$

We choose the domain

$$
I=\{x: x=0100 \vee x=0110 \vee \exists y[x=y 0100 \vee x=y 0110]\} .
$$

Clearly, $I$ contains 0100,0110 and is closed under $\circ$. We use $D_{1}$ when showing that $I$ is closed under $\circ$. We now proceed to show that the translation of each non-logical axiom of $\mathrm{D}+\forall x[x \preceq 1 \leftrightarrow x=1]$ is a theorem of $\mathrm{D}$.

By $\mathrm{D}_{1}$ and the definition of $\phi_{\circ}(x, y, z)$, the translation of $\mathrm{D}_{1}$ is a theorem of $\mathrm{D}$. The translation of $D_{2}$ is a theorem of $D$ since by $D_{2}$ we have

$$
x 0100=y 0100 \vee x 0110=y 0110 \Rightarrow x=y .
$$

The translation of $D_{3}$ is also a theorem of $D$ since by $D_{2}$ we have

$$
x 0100=y 0110 \Rightarrow x 010=y 011
$$

and $x 010=y 011$ contradicts $\mathrm{D}_{3}$.

We now show that the translation of $D_{4}$ is a theorem of $D$. We have

$$
\begin{array}{rlrl}
x \preceq 0100 & \Leftrightarrow x=0100 \vee x=010 \vee x \preceq 01 & & \left(\text { by } \mathrm{D}_{5}\right) \\
& \Leftrightarrow x=0100 \vee x=010 \vee x=01 \vee x \preceq 0 & \left(\text { by } \mathrm{D}_{6}\right) \\
& \Leftrightarrow x=0100 \vee x=010 \vee x=01 \vee x=0 \quad\left(\text { by } \mathrm{D}_{4}\right) .
\end{array}
$$

We need to show that we cannot have

$$
x=010 \vee x=01 \vee x=0 .
$$

Since $x \in I$, by the definition $I$, we have that $x=z 0100 \vee x=z 0110$ where $z$ could possibly be empty ( $x=z u$ with $z$ empty means $x=u$ since we do not have an empty string in D). We have

$$
\begin{aligned}
z 0100= & 010 \vee z 0100=01 \vee z 0100=0 \\
& \Downarrow \\
z 0100= & 010 \vee z 0100=01 \vee 11 z 0100=110 \\
& \Downarrow\left(\text { by } D_{2}\right) \\
z 010= & 01 \vee z 0100=01 \vee 11 z 010=11
\end{aligned}
$$


contradicting $\mathrm{D}_{3}$. We also have

$$
\begin{aligned}
z 0110= & 010 \vee z 0110=01 \vee z 0110=0 \\
& \Downarrow \\
0 z 0110= & 0010 \vee z 0110=01 \vee 00 z 0110=000 \\
& \Downarrow\left(\text { by } D_{2}\right) \\
0 z 01= & 00 \vee z 0100=01 \vee 00 z 011=00
\end{aligned}
$$

contradicting $\mathrm{D}_{3}$. Hence, $x \preceq 0100$ if and only if $x=0100$. Thus, the translation of $\mathrm{D}_{4}$ is a theorem of $\mathrm{D}$. By similar reasoning, the translation of $\forall x[x \preceq 1 \leftrightarrow x=1]$ is a theorem of $D$.

We now show that the translation of $D_{5}$ is a theorem of $D$. By $D_{5}$, we have

$$
x \preceq y 0100 \Leftrightarrow x=y 0100 \vee x=y 010 \vee x \preceq y 01 .
$$

By $\mathrm{D}_{6}$, we have

$$
x \preceq y 0100 \Leftrightarrow x=y 0100 \vee x=y 010 \vee x=y 01 \vee x \preceq y 0 .
$$

By $D_{5}$, we have

$$
x \preceq y 0100 \Leftrightarrow x=y 0100 \vee x=y 010 \vee x=y 01 \vee x=y 0 \vee x \preceq y .
$$

We need to show that we cannot have

$$
x=y 010 \vee x=y 01 \vee x=y 0 .
$$

Since $x, y \in I$, by the definition $I$, we have

$$
x=z 0100 \vee x=z 0110 \text { and } y=w 0100 \vee y=w 0110
$$

where $z$ and $w$ could possibly be empty. Reasoning as in the preceding paragraph shows that $x=y 010 \vee x=y 01 \vee x=y 0$ leads to a contradiction. Hence,

$$
x \preceq y 0100 \Leftrightarrow x=y 0100 \vee x \preceq y .
$$

Thus, the translation of $D_{5}$ is a theorem of $D$. By similar reasoning, the translation of $D_{6}$ is a theorem of $D$.

Since $\mathrm{D}$ proves the translation of each axiom of $\mathrm{D}+\forall x[x \preceq 1 \leftrightarrow x=1]$, it follows that $\mathrm{D}+\forall x[x \preceq 1 \leftrightarrow x=1]$ is interpretable in $\mathrm{D}$.

We now proceed to show that $\mathrm{Q}$ and $\mathrm{D}$ are mutually interpretable. We do this indirectly by showing that $\mathrm{D}$ is mutually interpretable with the theory BTQ which we have seen is mutually interpretable with $\mathrm{Q}$.

Theorem 6 BTQ is interpretable in D. 
Proof By Lemma 3, it suffices to show that BTQ is interpretable in $D+D_{4}^{\prime}$ where

$$
\mathrm{D}_{4}^{\prime} \equiv \forall x[x \preceq 1 \leftrightarrow x=1]
$$

We also observe that $\mathrm{D}$ proves the axioms $\mathrm{BTQ}_{1}, \mathrm{BTQ}_{2}$ and $\mathrm{BTQ}_{3}$ (the axioms are identical with $D_{1}, D_{2}, D_{3}$ ). So, to translate BTQ in $D+D_{4}^{\prime}$, we simply define a domain $K$ such that the axiom

$$
\mathrm{BTQ}_{4} \equiv \forall x[x=0 \vee x=1 \vee \exists y[x=y 0 \vee x=y 1]]
$$

holds restricted to $K$. Before defining $K$, we define auxiliary classes $A \supseteq B \supseteq C \supseteq$ $I \supseteq J \supseteq K$. We need to ensure that there is $y \in K$ such that $x=y 0$ or $x=y 1$ if $x \in K$ and $x \neq 0,1$. We do this by relying on $\preceq$. The idea is to first let $I$ be such that if $x \in I$ and $x \neq 0,1$; then we can find $y \preceq x$ such that $x=y 0$ or $x=y 1$. It will not necessarily be the case that $y \in I$. What we then do is to restrict $I$ so that we have a subclass $K$ that is downward closed under $\preceq$, that is, $x \in K$ and $y \preceq x$ implies $y \in K$. Since $K$ is a subclass of $I$, this immediately ensures that $\mathrm{BTQ}_{4}$ holds in $K$. We realize that in order for $I$ to be closed under $\circ$, it is useful if for all $x_{0}, x_{1} \in I$, we have that $x_{0} \preceq x_{0} x_{1}$ and that $y \preceq x_{1}$ implies $x_{0} y \preceq x_{0} x_{1}$. We therefore let $I$ be a subclass of a class $C$ with this property.

We let

$$
A=\{y: \forall x[x y \preceq x y]\} .
$$

By $\mathrm{D}_{5}$ and $\mathrm{D}_{6}$, we have $\mathrm{D} \vdash \forall x[x 0 \preceq x 0 \wedge x 1 \preceq x 1]$. Hence, $0,1 \in A$. We now show that $A$ is closed under o. Suppose $y_{0}, y_{1} \in A$. Since $y_{1} \in A$, we have

$$
\forall x\left[\left(x y_{0}\right) y_{1} \preceq\left(x y_{0}\right) y_{1}\right] .
$$

By $\mathrm{D}_{1}$, we then have $\forall x\left[x\left(y_{0} y_{1}\right) \preceq x\left(y_{0} y_{1}\right)\right]$. Hence, $y_{0} y_{1} \in A$. Thus, $A$ is closed under 0 .

We let

$$
B=\{x \in A: x \preceq x\}
$$

By $\mathrm{D}_{4}$ and $\mathrm{D}_{4}^{\prime}$, we have $\mathrm{D} \vdash 0 \preceq 0 \wedge 1 \preceq 1$. Hence, $0,1 \in B$. We now show that $B$ is closed under $\circ$. Suppose $x_{0}, x_{1} \in B$. Since $x_{1} \in B \subseteq A$, we have $x_{0} x_{1} \preceq x_{0} x_{1}$. Hence, $x_{0} x_{1} \in B$. Thus, $B$ is closed under $\circ$.

We let

$$
C=\{z \in B: \forall x y[x \preceq y z \leftrightarrow(x \preceq y \vee \exists u \preceq z[x=y u])]\} .
$$

By $D_{5}$ and $D_{4}$, we have

$$
\begin{aligned}
x \preceq y 0 & \Leftrightarrow x=y 0 \vee x \preceq y \\
& \Leftrightarrow x \preceq y \vee \exists u \preceq 0[x=y u] .
\end{aligned}
$$


Similarly, by $\mathrm{D}_{6}$ and $\mathrm{D}_{4}^{\prime}$, we have

$$
\begin{aligned}
x \preceq y 1 & \Leftrightarrow x=y 1 \vee x \preceq y \\
& \Leftrightarrow x \preceq y \vee \exists u \preceq 1[x=y u] .
\end{aligned}
$$

Hence, $0,1 \in C$. Next we show that $C$ is closed under o. Suppose $z_{0}, z_{1} \in C$. Then

$$
\begin{aligned}
x \preceq y\left(z_{0} z_{1}\right) & \Leftrightarrow x \preceq\left(y z_{0}\right) z_{1} & & \left(\mathrm{D}_{1}\right) \\
& \Leftrightarrow x \preceq y z_{0} \vee \exists u_{1} \preceq z_{1}\left[x=\left(y z_{0}\right) u_{1}\right] & & \left(z_{1} \in C\right) \\
& \Leftrightarrow x \preceq y \vee \exists u_{0} \preceq z_{0}\left[x=y u_{0}\right] \vee & & \\
& \Leftrightarrow x u_{1} \preceq z_{1}\left[x=\left(y z_{0}\right) u_{1}\right] & & \left(z_{0} \in C\right) \\
& \Leftrightarrow x \preceq y \vee \exists u_{0} \preceq z_{0}\left[x=y u_{0}\right] \vee & & \\
& \Leftrightarrow u_{1} \preceq z_{1}\left[x=y\left(z_{0} u_{1}\right)\right] & & \left(\mathrm{D}_{1}\right) \\
& \Leftrightarrow x \preceq y \vee \exists u \preceq z_{0} z_{1}[x=y u] & & \left(z_{1} \in C\right) .
\end{aligned}
$$

We justify the last equivalence as follows:

$(\Rightarrow)$ Suppose $u_{0} \preceq z_{0} \wedge x=y u_{0}$. Since $z_{1} \in C$ and $u_{0} \preceq z_{0}$, the right-left implication in the definition of $C$ tells us that $u_{0} \preceq z_{0} z_{1}$. We can thus let $u=u_{0}$. Suppose now $u_{1} \preceq z_{1} \wedge x=y\left(z_{0} u_{1}\right)$. Since $u_{1} \preceq z_{1}$ and $z_{1} \in C$, the right-left implication in the definition of $C$ tells us that $z_{0} u_{1} \preceq z_{0} z_{1}$. We can thus let $u=z_{0} u_{1}$.

$(\Leftarrow)$ Suppose $u \preceq z_{0} z_{1} \wedge x=y u$. Since $z_{1} \in C$, by the left-right implication in the definition of $C$, we have that $u \preceq z_{0}$ or there exists $u^{\prime} \preceq z_{1}$ such that $u=z_{0} u^{\prime}$. If $u \preceq z_{0}$, then $u_{0} \preceq z_{0} \wedge x=y u_{0}$ by setting $u_{0}=u$. If $u^{\prime} \preceq z_{1} \wedge u=z_{0} u^{\prime}$, then $u_{1} \preceq z_{1} \wedge x=y\left(z_{0} u_{1}\right)$ by setting $u_{1}=u^{\prime}$.

Hence, $z_{0} z_{1} \in C$. Thus, $C$ is closed under $\circ$.

We let

$$
I=\{x \in C: x=0 \vee x=1 \vee \exists y \preceq x[x=y 0 \vee x=y 1]\} .
$$

By definition of $I$, we have $0,1 \in I$. We now show that $I$ is closed under $\circ$. Suppose $x_{0}, x_{1} \in I$. Since $x_{1} \in I$, we have one of the following cases:

(1) $x_{1}=0 \vee x_{1}=1$

(2) $x_{1}=y_{1} 0 \vee x_{1}=y_{1} 1$ for some $y_{1} \preceq x_{1}$.

We first consider (1). Since $x_{0} \in C \subseteq B$, we have $x_{0} \preceq x_{0}$. Since $x_{1} \in C$, this implies $x_{0} \preceq x_{0} x_{1}$. Hence, $\exists y \preceq x_{0} x_{1}\left[x_{0} x_{1}=y 0 \vee x_{0} x_{1}=y 1\right]$. We now consider (2). We have $\exists y_{1} \preceq x_{1}\left[x_{0} x_{1}=\left(x_{0} y_{1}\right) 0 \vee x_{0} x_{1}=\left(x_{0} y_{1}\right) 1\right]$. Since $x_{1} \in C$, this implies

$$
\exists y \preceq x_{0} x_{1}\left[x_{0} x_{1}=y 0 \vee x_{0} x_{1}=y 1\right] .
$$

We thus see that both cases imply $x_{0} x_{1} \in I$. Hence, $I$ is closed under $\circ$.

We let

$$
J=\{v \in I: \forall x \preceq v[x \in I]\}
$$


By $\mathrm{D}_{4}$ and $\mathrm{D}_{4}^{\prime}$, we have $\mathrm{D} \vdash \forall x \preceq 0[x=0] \wedge \forall x \preceq 1[x=1]$. Hence, $0,1 \in J$. We now show that $J$ is closed under $\circ$. Suppose, $v_{0}, v_{1} \in J$ and $x \preceq v_{0} v_{1}$. Since $v_{1} \in C$, we have one of the following:

(a) $x \preceq v_{0}$

(b) $x=v_{0} y$ for some $y \preceq v_{1}$.

In case of $(a)$, we have $x \in I$ since $v_{0} \in J$. In case of $(b)$, we have $y \in I$ since $v_{1} \in J$. We also have $v_{0} \in I$ since $v_{0} \in J \subseteq I$. Since $I$ is closed under $\circ$, we then have $x=v_{0} y \in I$. We thus see that both cases imply $v_{0} v_{1} \in J$. Hence, $J$ is closed under $\circ$.

We let

$$
K=\{v \in J: \forall w \preceq v \forall x y[x \preceq y \wedge y \preceq w \rightarrow x \preceq w]\}
$$

By $\mathrm{D}_{4}$, we have

$$
x \preceq y \wedge y \preceq w \wedge w \preceq 0 \Rightarrow x=y=w=0 \preceq 0 .
$$

Hence, $0 \in K$. By $\mathrm{D}_{4}^{\prime}$, we have

$$
x \preceq y \wedge y \preceq w \wedge w \preceq 1 \Rightarrow x=y=w=1 \preceq 1
$$

Hence, $1 \in K$.

We now show that $K$ is closed under $\circ$. Suppose $v_{0}, v_{1} \in K$ and

$$
w \preceq v_{0} v_{1} \wedge x \preceq y \wedge y \preceq w
$$

We need to show that $x \preceq w$. From $w \preceq v_{0} v_{1}$ and $v_{1} \in K \subseteq C$, we have one of the following:

(I) $w \preceq v_{0}$

(II) $w=v_{0} u$ for some $u \preceq v_{1}$.

In case of (I), since $v_{0} \in K$, we have

$$
w \preceq v_{0} \wedge x \preceq y \wedge y \preceq w \Rightarrow x \preceq w
$$

In case of (II), we observe that $y \preceq w=v_{0} u$ and $u \preceq v_{1}$. Furthermore, since $v_{1} \in J$, we have that $u \preceq v_{1}$ implies $u \in I \subseteq C$. Then, $y \preceq w=v_{0} u$ and $u \in C$ implies that we have one of the following:

(IIa) $y \preceq v_{0}$

(IIb) $y=v_{0} u^{\prime}$ for some $u^{\prime} \preceq u$.

In case of (IIa), since $v_{0} \in K \subseteq B$, we have

$$
v_{0} \preceq v_{0} \wedge x \preceq y \wedge y \preceq v_{0} \Rightarrow x \preceq v_{0}
$$


since $v_{0} \in K$. Then, since $u \in C$, we have

$$
x \preceq v_{0} \Rightarrow x \preceq v_{0} u=w .
$$

In case of (IIb), we observe that $v_{1} \preceq v_{1}$ since $v_{1} \in K \subseteq B$. Then, $v_{1} \in K$ implies

$$
v_{1} \preceq v_{1} \wedge u^{\prime} \preceq u \wedge u \preceq v_{1} \Rightarrow u^{\prime} \preceq v_{1}
$$

Since $v_{1} \in K \subseteq J$, we have that $u^{\prime} \preceq v_{1}$ implies $u^{\prime} \in I \subseteq C$. Then, $x \preceq y=v_{0} u^{\prime}$ and $u^{\prime} \in C$ implies that we have one of the following:

(IIbi) $x \preceq v_{0}$

(IIbii) $x=v_{0} u^{\prime \prime}$ for some $u^{\prime \prime} \preceq u^{\prime}$.

In case of (IIbi), since $u \preceq v_{1}$ and $v_{1} \in K \subseteq J$ implies $u \in I \subseteq C$, we have

$$
x \preceq v_{0} \Rightarrow x \preceq v_{0} u=w .
$$

In case of (IIbii), we first observe that

$$
u \preceq v_{1} \wedge u^{\prime \prime} \preceq u^{\prime} \wedge u^{\prime} \preceq u \Rightarrow u^{\prime \prime} \preceq u
$$

since $v_{1} \in K$. Then, since $u \in C$, we have

$$
x=v_{0} u^{\prime \prime} \wedge u^{\prime \prime} \preceq u \Rightarrow x \preceq v_{0} u=w .
$$

We thus see that

$$
x \preceq y \wedge y \preceq w \wedge w \preceq v_{0} v_{1} \Rightarrow x \preceq w
$$

Hence, $v_{0} v_{1} \in K$. Thus, $K$ is closed under $\circ$.

We now show that the class $K$ has the following important property:

$$
\forall v \in K \forall w[w \preceq v \rightarrow w \in K]
$$

Indeed, suppose $v \in K$ and $w \preceq v$. We need to show that $w \in K$. By definition of $K$, we need to prove

$$
z \preceq w \wedge x \preceq y \wedge y \preceq z \Rightarrow x \preceq z .
$$

Since $v \in K \subseteq B$, we have $v \preceq v$. Then,

$$
v \in K \wedge v \preceq v \wedge z \preceq w \wedge w \preceq v \Rightarrow z \preceq v .
$$

This in turn implies

$$
v \in K \wedge z \preceq v \wedge x \preceq y \wedge y \preceq z \Rightarrow x \preceq z
$$


Hence, $w \in K$. Thus, $(*)$ holds.

We are now ready to give an interpretation of BTQ in D. We choose the domain $K$. We translate 0,1 and $\circ$ as

$$
\begin{aligned}
\phi_{0}(x) & \equiv x=0 \\
\phi_{1}(x) & \equiv x=1 \\
\phi_{\circ}(x, y, z) & \equiv z=x y .
\end{aligned}
$$

It is clear that the translations of $\mathrm{BTQ}_{1}, \mathrm{BTQ}_{2}$ and $\mathrm{BTQ}_{3}$ are theorems of $\mathrm{D}$. We now show that the translation of $\mathrm{BTQ}_{4}$ is a theorem of D. Let $x \in K$. Since $K \subseteq I$, we have

$$
x=0 \vee x=1 \vee \exists y \preceq x[x=y 0 \vee x=y 1] .
$$

If the third disjunct is the case, then we have $y \in K$ by $(*)$. Thus, the translation of $\mathrm{BTQ}_{4}$ is a theorem of D. Hence, BTQ is interpretable in D.

Theorem 7 D is interpretable in BTQ.

Proof We choose the domain $J(x) \equiv x=x$. We translate 0,1 , ० and and $\preceq$ as follows:

$$
\begin{aligned}
\phi_{0}(x) & \equiv x=0 \\
\phi_{1}(x) & \equiv x=1 \\
\phi_{\circ}(x, y, z) & \equiv z=x y \\
\phi_{\preceq}(x, y) & \equiv y=x \vee \exists z[y=x z] .
\end{aligned}
$$

It is clear that the translation of $D_{1}, D_{2}$ and $D_{3}$ are theorems of BTQ. We now show that the translation of

$$
\mathrm{D}_{4} \equiv \forall x[x \preceq 0 \leftrightarrow x=0]
$$

is a theorem of BTQ. So, suppose $0=x \vee \exists z$ [ $0=x z$ ]. It is not difficult to see that we cannot have $\exists z[0=x z]$. Hence, the translation of $\mathrm{D}_{4}$ is a theorem of BTQ.

We now show that the translation of

$$
\mathrm{D}_{5} \equiv \forall x y[x \preceq y 0 \leftrightarrow(x=y 0 \vee x \preceq y)]
$$

is a theorem of BTQ. We need to show that

$$
y 0=x \vee \exists z[y 0=x z] \Leftrightarrow y 0=x \vee y=x \vee \exists u[y=x u] .
$$

It suffices to show that

$$
\exists z[y 0=x z] \Leftrightarrow y=x \vee \exists u[y=x u] .
$$


The right-left implication of $(*)$ is trivial. The left-right implication holds since

$$
\begin{aligned}
& y 0=x z \Rightarrow(y 0=x 0 \wedge z=0) \vee \\
& \exists z^{\prime}\left[y 0=x\left(z^{\prime} 0\right) \wedge z=z^{\prime} 0\right] \text { (by } \mathrm{BTQ}_{4}, \mathrm{BTQ}_{3} \text { ) } \\
& \Rightarrow(y 0=x 0 \wedge z=0) \vee \\
& \exists z^{\prime}\left[y 0=\left(x z^{\prime}\right) 0 \wedge z=z^{\prime} 0\right] \quad\left(\text { by } \mathrm{BTQ}_{1}\right) \\
& \Rightarrow(y=x \wedge z=0) \vee \\
& \exists z^{\prime}\left[y=x z^{\prime} \wedge z=z^{\prime} 0\right] \quad \text { (by } \mathrm{BTQ}_{2} \text { ) } \\
& \Rightarrow y=x \vee \exists u[y=x u] \text {. }
\end{aligned}
$$

Thus, the translation of $\mathrm{D}_{5}$ is a theorem of BTQ.

By similar reasoning, the translation of $D_{6}$ is a theorem of BTQ.

Thus, D is interpretable in BTQ.

\section{Minimality results}

This section is devoted to show that the axiomatizations of WD, WBT and BTQ are minimal essentially undecidable, which is to say that removing any one of the axioms (axiom schemas) gives a theory that is not essentially undecidable. We are not able to show that our axiomatization of $D$ is minimal essentially undecidable, but we reduce the problem to showing that $\mathrm{D}-\mathrm{D}_{5}$ and $\mathrm{D}-\mathrm{D}_{6}$ are not essentially undecidable. However, as $D$ has a finite axiomatization, we can make it minimal essentially undecidable by replacing some of the axioms with their conjunction.

We now proceed to show that $W B T-W B T_{1}, W D-W_{1}$ and $D-D_{1}$ are not essentially undecidable by interpreting them in S2S. S2S is a monadic second order theory whose language is $\left\{e, 0,1, S_{0}, S_{1}\right\}$, where $e, 0$ and 1 are constant symbols and $S_{0}$ and $S_{1}$ are unary function symbols. The axioms of S2S are the true sentences in the standard second-order structure where the universe is $\{0,1\}^{*}$. The symbol e is interpreted as the empty string, 0 is interpreted as 0 and 1 is interpreted as 1 . The function symbol $\mathrm{S}_{0}$ is interpreted as the function that takes a bit string and concatenates it with the bit 0 , and the function symbol $S_{1}$ is interpreted as the function that takes a bit string and concatenates it with the bit 1 . We have quantifiers that range over $\{0,1\}^{*}$, and we have quantifiers that range over subsets of $\{0,1\}^{*}$. It was proved in Rabin [13] that S2S is decidable. Our interpretation of WBT-WBT 1 in S2S does not use the monadic second order part, and this makes the induced algorithm more efficient. It is known that extending S2S with the prefix relation does not change the expressive power of S2S (see Börger et al. [1] p. 317). We also show this when we interpret WD-WD and $D-D_{1}$ in $S 2 S$.

We recall that biterals are associated to the left. So, $((0 \circ 0) \circ 0)$ is a biteral while $(0 \circ(0 \circ 0))$ is not. Although we have so far not needed to take this into account, it now becomes important.

Lemma $4 W B T-W B T_{1}$ and $W D-W D_{1}$ are not essentially undecidable. 
Proof We interpret the two theories in S2S as follows: We choose the domain $\delta(x) \equiv$ $x=x$. We map 0 and 1 to

$$
\begin{aligned}
& \phi_{0}(x) \equiv x=0 \\
& \phi_{1}(x) \equiv x=1 .
\end{aligned}
$$

So, 0 and 1 are realized as 0 and 1 , respectively. We map o to

$$
\begin{aligned}
\phi_{\circ}(x, y, z) \equiv & ((x=\mathrm{e} \vee(y \neq 0 \wedge y \neq 1)) \wedge z=\mathrm{e}) \vee \\
& {\left[x \neq \mathrm{e} \wedge\left(\left(y=0 \wedge z=\mathrm{S}_{0} x\right) \vee\right.\right.} \\
& \left.\left.\left(y=1 \wedge z=\mathrm{S}_{1} x\right)\right)\right] .
\end{aligned}
$$

This means that $\circ$ is realized as the function

$$
(x, y) \mapsto \begin{cases}\varepsilon & \text { if } x=\varepsilon \text { or } y \notin\{0,1\} \\ x 0 & \text { if } x \neq \varepsilon \text { and } y=0 \\ x 1 & \text { if } x \neq \varepsilon \text { and } y=1 .\end{cases}
$$

Recalling that biterals are associated to the left, it is clear that the translation of each instance of $W_{B T}$ and $W_{2}$ is a theorem of $S 2 S$. We now show that the translation of each instance of

$$
\mathrm{WBT}_{3} \equiv \forall x\left[x \sqsubseteq_{\mathrm{s}} \bar{\alpha} \rightarrow \bigvee_{\gamma \in\langle\ldots \alpha\rangle} x=\bar{\gamma}\right]
$$

is a theorem of S2S. We recall that

$$
x \sqsubseteq s y \equiv y=x \vee \exists u v[y=u x \vee y=x v \vee y=(u x) v \vee y=u(x v)] .
$$

Let $\alpha \neq \varepsilon$. Suppose $\beta$ is a substring of $\alpha$ w.r.t. $\phi_{\circ}(x, y, z)$. We show that $\beta \neq \varepsilon$ and that $\beta$ is a substring of $\alpha$ in the actual sense. We have one of the following cases:

$-\alpha=\beta$

- There exists $u$ such that $\phi_{\circ}(u, \beta, \alpha)$. Then, $\alpha=u \beta, \beta \in\{0,1\}$ and $u \neq \varepsilon$.

- There exists $v$ such that $\phi_{\circ}(\beta, v, \alpha)$. Then, $\alpha=\beta v, \beta \neq \varepsilon$ and $v \in\{0,1\}$.

- There exist $u, v$ and $w$ such that $\phi_{\circ}(u, \beta, w) \wedge \phi_{\circ}(w, v, \alpha)$. Then, $\alpha=u \beta v, u \neq \varepsilon$ and $\beta, v \in\{0,1\}$.

- There exist $u, v$ and $w$ such that $\phi_{\circ}(\beta, v, w) \wedge \phi_{\circ}(u, w, \alpha)$. This is not possible since $\phi_{\circ}(u, w, \alpha)$ implies $w \in\{0,1\}$ while we cannot have $v$ such that $\phi_{\circ}(\beta, v, 0)$ or $\phi_{\circ}(\beta, v, 1)$.

We thus see that $\beta$ is a substring of $\alpha$ in the actual sense.

We map $\preceq$ to

$$
\phi_{\preceq}(x, y) \equiv \exists Y\left[\mathrm{e} \notin Y \wedge x \in Y \wedge y \in Y \wedge \mathrm{S}_{0} y \notin Y \wedge \mathrm{S}_{1} y \notin Y \wedge\right.
$$




$$
\begin{aligned}
& \forall z\left[\left(z \neq \mathrm{e} \wedge\left(\mathrm{S}_{0} z \in Y \vee \mathrm{S}_{1} z \in Y\right)\right) \rightarrow z \in Y\right] \wedge \\
& \left.\forall w\left[\mathrm{~S}_{0} w \in Y \rightarrow \mathrm{S}_{1} w \notin Y\right]\right] .
\end{aligned}
$$

This formula forces $\preceq$ to be realized as the prefix relation on $\{0,1\}^{+}$. Indeed, suppose $\alpha, \beta \in\{0,1\}^{+}$and $\alpha$ is related to $\beta$ as defined by $\phi_{\preceq}(x, y)$. The first line in $\phi_{\preceq}(x, y)$ tells us that there exists $Y \subseteq\{0,1\}^{+}$such that

$$
\alpha, \beta \in Y \quad \text { and } \quad \beta 0, \beta 1 \notin Y \text {. }
$$

The second line in $\phi_{\preceq}(x, y)$ tells us that if $\gamma \in Y$, then all the non-empty prefixes of $\gamma$ are also in $Y$. Let

$$
\{0,1\} \ni \alpha_{0} \prec \alpha_{1} \prec \ldots \prec \alpha_{k}=\alpha \quad \text { and } \quad\{0,1\} \ni \beta_{0} \prec \beta_{1} \prec \ldots \prec \beta_{m}=\beta
$$

denote all the prefixes of $\alpha$ and $\beta$. The notation $\gamma \prec \delta$ then means that $\gamma$ is the longest proper prefix of $\delta$. We show by induction that for each $0 \leq j \leq k$, there exists $0 \leq i \leq m$ such that $\alpha_{j}=\beta_{i}$, which implies that $\alpha$ is a prefix of $\beta$. The third line in $\phi_{\preceq}(x, y)$ tells us that exactly one of 0 and 1 belongs to $Y$ since we get for free that $\forall w\left[\mathrm{~S}_{1} w \in Y \rightarrow \mathrm{S}_{0} w \notin Y\right]$. Hence, $\alpha_{0}=\beta_{0}$. Suppose now that for $0 \leq j<k$ there exists $0 \leq i \leq m$ such that $\alpha_{j}=\beta_{i}$. We observe that we must have that $i<m$ since $\alpha_{j}=\beta_{i}=\beta$ and $\alpha_{j+1} \in Y$ would contradict $\beta 0, \beta 1 \notin Y$. It remains to show that $\alpha_{j+1}=\beta_{i+1}$. The third line in $\phi_{\preceq}(x, y)$ tells us that exactly one of $\alpha_{j} 0$ and $\alpha_{j} 1$ belongs to $Y$. Hence, $\alpha_{j+1}=\beta_{i+1}$. Thus, by induction, each prefix of $\alpha$ is a prefix of $\beta$.

We thus see that this translation has the desired properties. This translation shows us how to define decidable models of the theories in question.

Lemma $5-D_{1}$ is not essentially undecidable.

Proof We modify the translation in Lemma 4 as follows: We map o to

$$
\begin{aligned}
\phi_{\circ}(x, y, z) \equiv & ((y \neq 0 \vee y \neq 1) \wedge z=x) \vee\left(y=0 \wedge z=\mathrm{S}_{0} x\right) \vee(y=1 \\
& \left.\wedge z=\mathrm{S}_{1} x\right) .
\end{aligned}
$$

This means that $\circ$ is realized as the function

$$
(x, y) \mapsto \begin{cases}x & \text { if } y \notin\{0,1\} \\ x 0 & \text { if } y=0 \\ x 1 & \text { if } y=1 .\end{cases}
$$

We need this modification to ensure that the axiom $\mathrm{D}_{3} \equiv \forall x y[x 0 \neq y 1]$ holds.

We observe that the simple translation of o we give in the preceding lemma does not work in the case of Lemma 4 since the axiom scheme $\mathrm{WBT}_{3}$ then fails. Indeed, for any non-empty bit string $\alpha$ and any bit string $\beta \neq 0$, 1 , we have $\phi_{\circ}(\bar{\alpha}, \bar{\beta}, \bar{\alpha})$. This means that all substring different from 0 and 1 are substrings of $\alpha$ w.r.t. $\phi_{\circ}(x, y, z)$. 
Lemma $6 B T Q-B T Q_{1}$ is not essentially undecidable.

Proof Let $\Sigma$ denote the set of all sentences in first order logic with equality and the language $\{0,1,+, \times, \leq\}$ true in the structure $(\mathbb{R}, 0,1,+, \times, \leq)$. It was shown by Tarski [17] that $\Sigma$ is decidable. We show that BTQ $-\mathrm{BTQ}_{1}$ is interpretable in $\Sigma$.

We now define a relative interpretation of $\mathrm{BTQ}-\mathrm{BTQ}_{1}$ in $\Sigma$. We relativize quantification to $\lambda(x): \equiv x=x$ and use the following translation of symbols

$$
\begin{aligned}
& 0 \mapsto \psi_{0}(x): \equiv x=0 \\
& 1 \mapsto \psi_{1}(x): \equiv x=1 \\
& \circ \mapsto \psi_{\circ}(x, y, z)
\end{aligned}
$$

where $\psi_{\circ}(x, y, z)$ is a formula which defines in $(\mathbb{R}, 0,1,+, \times, \leq)$ the function $h=$ $h_{3} \circ h_{2} \circ h_{1}: \mathbb{R}^{2} \rightarrow \mathbb{R}$ where

$$
\begin{aligned}
h_{1}(r, u) & = \begin{cases}-2+\frac{2 r}{1+\sqrt{1+4 r^{2}}} & \text { if } u=0 \\
\frac{2 r}{1+\sqrt{1+4 r^{2}}} & \text { if } u=1 \\
0 & \text { otherwise }\end{cases} \\
h_{2}(r) & = \begin{cases}\frac{1+r}{2} & \text { if } r \in(-3,-1) \cup(-1,1) \\
0 & \text { otherwise }\end{cases} \\
h_{3}(r) & = \begin{cases}\frac{1}{r}-1 & \text { if } r \in(0,1) \\
\frac{1}{r}+1 & \text { if } r \in(-1,0) \\
0 & \text { otherwise } .\end{cases}
\end{aligned}
$$

We first observe that the map $\mathbb{R} \rightarrow(-1,1)$ defined by

$$
r \mapsto \frac{2 r}{1+\sqrt{1+4 r^{2}}}
$$

is a bijection. The sections $h(-, 0)$ and $h(-, 1)$ are injective and have images $(-\infty, 0)$ and $(0, \infty)$. This is because we have the following sequences of bijections:

$$
\begin{aligned}
& \mathbb{R} \underset{h_{1}(-, 0)}{\stackrel{\sim}{\sim}}(-3,-1) \underset{h_{2}}{\stackrel{\sim}{\longrightarrow}}(-1,0) \underset{h_{3}}{\stackrel{\sim}{\longrightarrow}}(-\infty, 0) \\
& \mathbb{R} \underset{h_{1}(-, 1)}{\stackrel{\sim}{\longrightarrow}}(-1,1) \underset{h_{2}}{\stackrel{\sim}{\longrightarrow}}(0,1) \underset{h_{3}}{\stackrel{\sim}{\longrightarrow}}(0, \infty) .
\end{aligned}
$$

The fact that $h(-, 0)$ and $h(-, 1)$ are injective and have disjoint images implies that the translation of

$$
\mathrm{BTQ}_{2} \equiv \forall x y[x \neq y \rightarrow(x 0 \neq y 0 \wedge x 1 \neq y 1)]
$$


and

$$
\mathrm{BTQ}_{3} \equiv \forall x y[x 0 \neq y 1]
$$

are theorems of $\Sigma$. The translation of

$$
\mathrm{BTQ}_{4} \equiv \forall x[x=0 \vee x=1 \vee \exists y[x=y 0 \vee x=y 1]]
$$

is also a theorem of $\Sigma$ since we have defined $\psi_{0}(x) \equiv x=0$ and since the union of the images of $h(-, 0)$ and $h(-, 1)$ is

$$
(-\infty, 0) \cup(0, \infty)=\mathbb{R} \backslash\{0\}
$$

The translation shows how to define a decidable model of $B T Q-B T Q_{1}$.

One of the referees has observed that we get a simpler proof of the preceding lemma by considering the translation given in Lemma 4 and restricting the domain to the set of all non-empty strings. We can thus for example translate $\circ$ as follows

$$
(x, y) \mapsto \begin{cases}x 0 & \text { if } y=0 \\ x 1 & \text { if } y=1 \\ 0 & \text { otherwise. }\end{cases}
$$

Lemma $7 W B T-W B T_{2}$ and $W D-W D_{2}$ are not essentially undecidable.

Proof We obtain a one element model $\mathfrak{A}$ of $W B T-W_{2}$ and $W D-W_{2}$ as follows:

- the universe is $\{0\}$

$-0^{\mathfrak{A}}=0,1^{\mathfrak{A}}=0$

$-0 \circ^{\mathfrak{A}} 0=0$

$-\preceq^{\mathfrak{A}}=\{(0,0)\}$.

Since $\circ^{\mathfrak{A}}$ is associative, $\mathrm{WBT}_{1}$ and $\mathrm{WD}_{1}$ hold in $\mathfrak{A}$. We observe that $\mathfrak{A}$ satisfies

$$
\forall x\left[x \sqsubseteq_{s} \bar{\alpha} \rightarrow x=\bar{\alpha}\right] \text { and } \forall x\left[x \preceq \bar{\alpha} \leftrightarrow \bigvee_{\beta \preceq^{\mathfrak{D} \alpha}} x=\bar{\beta}\right]
$$

since there is only one element in the universe. Thus, $\mathrm{WBT}_{3}$ and $\mathrm{WD}_{3}$ hold in $\mathfrak{A}$.

Lemma $8 B T Q-B T Q_{2}$ is not essentially undecidable.

Proof We obtain a two element model $\mathfrak{A}$ of $\mathrm{BTQ}-\mathrm{BTQ}_{2}$ as follows:

- the universe is $\{0,1\}$

$-0^{\mathfrak{A}}=0,1^{\mathfrak{A}}=1$

$-\forall x, y \in\{0,1\}(x \circ \mathfrak{A} y=y)$. 
The operator $\circ^{\mathfrak{A}}$ is clearly associative. The axiom $\forall x y[x 0 \neq y 1]$ holds since

$$
x \circ^{\mathfrak{A}} 0=0 \neq 1=y \circ^{\mathfrak{A}} 1 .
$$

The axiom $\forall x[x=0 \vee x=1 \vee \exists y[x=y 0 \vee x=y 1]]$ holds since the universe is $\left\{0^{\mathfrak{A}}, 1^{\mathfrak{A}}\right\}$.

The following proof of the next Lemma was suggested by one of the referees . We show that $D-D_{2}$ is not essentially undecidable by interpreting it in Presburger Arithmetic. Presburger Arithmetic refers to all sentences in first-order logic with equality and the language $\{0,1,+,<\}$ true in the structure $(\mathbb{N}, 0,1,+,<)$. It is shown that Presburger Arithmetic is decidable in Chapter 3.4 of Smoryński [15]. The idea is to consider the free monoid generated by 0 and 1 modulo the equations $00=0$ and $11=1$. The universe of this structure then consists of strings of the form $(01)^{n}$, $1(01)^{n},(01)^{n} 0,1(01)^{n} 0$, with $n$ being a natural number. Since we do not have the empty string, we have $n>0$ when the string is of the form $(01)^{n}$. The concatenation operator on this set is described as follows

$$
x \star y= \begin{cases}x y & \text { if } x=u a, y=b v, a \neq b \text { and } a, b \in\{0,1\} \\ u a v & \text { if } x=u a, y=a v \text { and } a \in\{0,1\} .\end{cases}
$$

Observe that $u$ and $v$ denote the empty string in the cases $x, y \in\{0,1\}$. It is clear that axiom $\mathrm{D}_{3} \equiv \forall x y[x 0 \neq x 1]$ holds in this model. It also follows easily from the definition of $\star$ that $(x \star y) \star z=x \star(y \star z)$ when $y$ has length at least two. To see that $\star$ is indeed associative, we observe that given distinct $a, b \in\{0,1\}$, we have

$$
\begin{aligned}
(x \star a) \star z & = \begin{cases}x a z & \text { if } x=u b, z=b v \\
x a v & \text { if } x=u b, z=a v \\
u a z & \text { if } x=u a, z=b v \\
u a v & \text { if } x=u a, z=a v\end{cases} \\
& =x \star(a \star z) .
\end{aligned}
$$

Thus, axiom $\mathrm{D}_{1} \equiv \forall x y z[(x y) z=x(y z)]$ holds.

We interpret $\preceq$ as the prefix relation, i.e., $x \preceq y$ if and only if $x=y$ or there exists $z$ such that $y=x \star z$. Axiom $\mathrm{D}_{4} \equiv \forall x[x \preceq 0 \leftrightarrow x=0]$ holds since $x \star z=0$ implies $x=z=0$. Axiom $\mathrm{D}_{5} \equiv \forall x y[x \preceq y 0 \leftrightarrow(x=y 0 \vee x \preceq y]$ holds by the following reasoning:

- Suppose $x \preceq y \star 0$ and $y \star 0=x \star z$ for some $z$. If $y$ has 0 as a suffix, then $y=x \star z$, which implies $x \preceq y$. Assume now $y$ has 1 as a suffix. We have that $z=0$ or $z=u 10$ where $u$ is possibly empty. We first assume $z=0$. If $x$ has 0 as a suffix, then $y \star 0=x$. If $y$ and $x$ both have 1 as suffices, then $y=x$, which in turn implies $x \preceq y$. Suppose now $z=u 10$. Then $y=x \star u 1$, which in turn implies $x \preceq y$.

- Suppose $x=y \star 0 \vee y=x \vee \exists z[y=x \star z]$. Then, by associativity of $\star$, we have $x=y \star 0 \vee y \star 0=x \star 0 \vee \exists z[y \star 0=x \star(z \star 0)]$. Hence $x \preceq y \star 0$. 
By similar reasoning, axiom $\mathrm{D}_{6} \equiv \forall x y[x \preceq y 1 \leftrightarrow(x=y 1 \vee x \preceq y]$ holds.

Now, to interpret $D-D_{2}$ in Presburger Arithmetic, we associate strings with natural numbers as follows

$$
(01)^{n} \mapsto 4 n, \quad 1(01)^{n} \mapsto 4 n+1, \quad(01)^{n} 0 \mapsto 4 n+2, \quad 1(01)^{n} 0 \mapsto 4 n+3 .
$$

It is then not difficult to see that $\star$ is definable in Presburger Arithmetic. This in turn implies that the prefix relation is definable. We thus have the following result.

Lemma $9 D-D_{2}$ is not essentially undecidable.

Lemma $10 B T Q-B T Q_{3}$ and $D-D_{3}$ are not essentially undecidable.

Proof Since Presburger Arithmetic is decidable, we obtain a decidable model of $\mathrm{BTQ}-\mathrm{BTQ}_{3}$ and $\mathrm{D}-\mathrm{D}_{3}$ as follows:

- the universe is the set of natural numbers $\mathbb{N}$

$-0^{\mathfrak{A}}=0,1^{\mathfrak{A}}=0$

$-\forall x, y \in \mathbb{N}\left(x \circ^{\mathfrak{A}} y=x+y\right)$

$-x \preceq^{\mathfrak{A}} y \Leftrightarrow x=y$.

It is not difficult to see that axioms of $B T Q-B^{2} Q_{3}$ hold. Axiom

$$
\mathrm{D}_{4} \equiv \forall x[x \preceq 0 \leftrightarrow x=0]
$$

holds since $x \preceq^{\mathfrak{A}} 0^{\mathfrak{A}}$ if and only if $x=0^{\mathfrak{A}}$. The axioms

$$
\begin{aligned}
& \mathrm{D}_{5} \equiv \forall x y[x \preceq y 0 \leftrightarrow(x=y 0 \vee x \preceq y)] \text { and } \\
& \mathrm{D}_{6} \equiv \forall x y[x \preceq y 1 \leftrightarrow(x=y 1 \vee x \preceq y)]
\end{aligned}
$$

hold since $x \preceq^{\mathfrak{A}} x$ and $y \circ^{\mathfrak{A}} 1^{\mathfrak{A}}=y=y \circ^{\mathfrak{A}} 0^{\mathfrak{A}}$.

Lemma $11 W D-W D_{3}, W B T-W B T_{3}, B T Q-B T Q_{4}$ and $D-D_{4}$ are not essentially undecidable.

Proof We start by showing that $\mathrm{BTQ}-\mathrm{BTQ}_{4}$ has a decidable model. We consider the set $\mathrm{M}_{2}\left(\mathbb{R}_{\geq 0}\right)$ of $2 \times 2$ matrices with coefficients in $\mathbb{R}_{\geq 0}$ and which are such that the first entry is strictly positive. So, the elements of $M_{2}\left(\mathbb{R}_{\geq 0}\right)$ are of the form

$$
\left(\begin{array}{ccc}
x \in \mathbb{R}_{>0} & y \in \mathbb{R}_{\geq 0} \\
z \in \mathbb{R}_{\geq 0} & w \in \mathbb{R}_{\geq 0}
\end{array}\right) .
$$

We consider the binary operation of matrix multiplication, denoted $\times$. It is not difficult to verify that $M_{2}\left(\mathbb{R}_{\geq 0}\right)$ is closed under matrix multiplication. We can consider this structure as a first-order structure for the language $\left\{c_{0}, c_{1}, \times\right\}$ where $c_{0}$ and $c_{1}$ are constant symbols for the matrices

$$
\left(\begin{array}{ll}
1 & 0 \\
1 & 1
\end{array}\right) \text { and }\left(\begin{array}{ll}
1 & 1 \\
0 & 1
\end{array}\right)
$$


respectively. Let $\operatorname{Th}\left(\mathrm{M}_{2}\left(\mathbb{R}_{\geq 0}\right)\right)$ denote the set of sentences true in this structure. We show that $B T Q-B T Q_{4}$ is interpretable in $\operatorname{Th}\left(M_{2}\left(\mathbb{R}_{\geq 0}\right)\right)$ and that $\operatorname{Th}\left(M_{2}\left(\mathbb{R}_{\geq 0}\right)\right)$ is decidable. Since relative interpretability preserves the property of being essentially undecidable, this means that $\mathrm{BTQ}-\mathrm{BTQ}_{4}$ is not essentially undecidable.

We interpret $B T Q-B^{2} Q_{4}$ in $\operatorname{Th}\left(M_{2}\left(\mathbb{R}_{\geq 0}\right)\right)$ as follows:

- We choose the domain $\delta(x) \equiv x=x$.

- We map 0 to $\phi_{0}(x)=x=c_{0}$.

- We map 1 to $\phi_{1}(x) \equiv x=c_{1}$.

- We map o to $\phi_{\circ}(x, y, z) \equiv x \times y=z$.

Since matrix multiplication is associative, the translation of

$$
\mathrm{BTQ}_{1} \equiv \forall x y z[(x y) z=x(y z)]
$$

is a theorem of $\operatorname{Th}\left(M_{2}\left(\mathbb{R}_{\geq 0}\right)\right)$. To see that the translation of

$$
\mathrm{BTQ}_{2} \equiv \forall x y[x \neq y \rightarrow(x 0 \neq y 0 \wedge x 1 \neq y 1)]
$$

is a theorem of $\operatorname{Th}\left(M_{2}\left(\mathbb{R}_{\geq 0}\right)\right)$, we observe that

$$
\begin{aligned}
\left(\begin{array}{ll}
x & y \\
z & w
\end{array}\right) \times\left(\begin{array}{ll}
1 & 0 \\
1 & 1
\end{array}\right)=\left(\begin{array}{ll}
a & b \\
c & d
\end{array}\right) \times\left(\begin{array}{ll}
1 & 0 \\
1 & 1
\end{array}\right) & \Rightarrow\left(\begin{array}{ll}
x+y & y \\
z+w & w
\end{array}\right)=\left(\begin{array}{ll}
a+b & b \\
c+d & d
\end{array}\right) \\
& \Rightarrow x=a \wedge y=b \wedge z=c \wedge w=d
\end{aligned}
$$

and

$$
\begin{aligned}
\left(\begin{array}{ll}
x & y \\
z & w
\end{array}\right) \times\left(\begin{array}{ll}
1 & 1 \\
0 & 1
\end{array}\right)=\left(\begin{array}{ll}
a & b \\
c & d
\end{array}\right) \times\left(\begin{array}{ll}
1 & 1 \\
0 & 1
\end{array}\right) & \Rightarrow\left(\begin{array}{ll}
x & x+y \\
z & z+w
\end{array}\right)=\left(\begin{array}{ll}
a & a+b \\
c & c+d
\end{array}\right) \\
& \Rightarrow x=a \wedge y=b \wedge z=c \wedge w=d .
\end{aligned}
$$

To see that translation of

$$
\mathrm{BTQ}_{3} \equiv \forall x y[x 0 \neq y 1]
$$

is a theorem of $\operatorname{Th}\left(M_{2}\left(\mathbb{R}_{\geq 0}\right)\right)$, we observe that

$$
\begin{aligned}
\left(\begin{array}{ll}
x & y \\
z & w
\end{array}\right) \times\left(\begin{array}{ll}
1 & 0 \\
1 & 1
\end{array}\right)=\left(\begin{array}{ll}
a & b \\
c & d
\end{array}\right) \times\left(\begin{array}{ll}
1 & 1 \\
0 & 1
\end{array}\right) & \Rightarrow\left(\begin{array}{ll}
x+y & y \\
z+w & w
\end{array}\right)=\left(\begin{array}{ll}
a & a+b \\
c & c+d
\end{array}\right) \\
& \Rightarrow x+y=a \wedge y=a+b \\
& \Rightarrow x+a+b=a \\
& \Rightarrow x+b=0 \\
& \Rightarrow x=0 .
\end{aligned}
$$


But we cannot have $x=0$ by how the set $M_{2}\left(\mathbb{R}_{\geq 0}\right)$ is defined. We then see that the translation of $\mathrm{BTQ}_{3}$ is a theorem of $\operatorname{Th}\left(\mathrm{M}_{2}\left(\mathbb{R}_{\geq 0}\right)\right)$

To see that $\operatorname{Th}\left(\mathrm{M}_{2}\left(\mathbb{R}_{\geq 0}\right)\right)$ is decidable, we use Tarski's result that the set $\operatorname{Th}(\mathbb{R})$ of sentences which are true in the ordered field of real numbers $(\mathbb{R}, 0,1,+, \times, \leq)$ is decidable (see Tarski [17]). To see that this is the case, we associate each sentence $\phi$ in the language of $\operatorname{Th}\left(M_{2}\left(\mathbb{R}_{\geq 0}\right)\right)$ with a sentence $\phi^{*}$ in the language of $T h(\mathbb{R})$ such that

$$
\phi \in \operatorname{Th}\left(\mathrm{M}_{2}\left(\mathbb{R}_{\geq 0}\right)\right) \Leftrightarrow \phi^{*} \in \operatorname{Th}(\mathbb{R})
$$

We start by associating $c_{0}$ and $c_{1}$ with

$$
\left(\begin{array}{ll}
1 & 0 \\
1 & 1
\end{array}\right) \text { and }\left(\begin{array}{ll}
1 & 1 \\
0 & 1
\end{array}\right)
$$

respectively, and by associating each variable $x$ with

$$
\left(\begin{array}{ll}
x_{1} & x_{2} \\
x_{3} & x_{4}
\end{array}\right)
$$

We recall that matrix multiplication is defined by

$$
\left(\begin{array}{ll}
x & y \\
z & w
\end{array}\right) \times\left(\begin{array}{ll}
a & b \\
c & d
\end{array}\right)=\left(\begin{array}{cc}
x a+y c & x b+y d \\
z a+w c & z b+w d
\end{array}\right) .
$$

By following this definition, we associate each term $s$ in the language of $\operatorname{Th}\left(M_{2}\left(\mathbb{R}_{\geq 0}\right)\right)$ with a matrix

$$
\left(\begin{array}{ll}
p_{s, 1} & p_{s, 2} \\
p_{s, 3} & p_{s, 4}
\end{array}\right)
$$

where each $p_{s, i}$ is a term in the language of Th $(\mathbb{R})$. So, for example, the term $x \times y$ is associated with the matrix

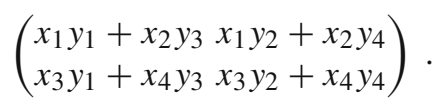

Next we define $\phi^{*}$ by recursion on the structure of $\phi$ as follows:

(1) If $\phi \equiv s=t$, then

$$
\phi^{*} \equiv \bigwedge_{x \in S \cup T, 1 \leq i \leq 4} x_{i} \geq 0 \wedge \bigwedge_{y \in S \cup T} y_{1} \neq 0 \wedge \bigwedge_{i=1}^{4} p_{s, i}=p_{t, i}
$$

where $S$ and $T$ denotes the set of variables occurring in $s$ and $t$, respectively.

(2) If $\phi \equiv \neg \alpha$, then $\phi^{*} \equiv \neg \alpha^{*}$ 
(3) If $\phi \equiv \alpha \vee \beta$, then $\phi^{*} \equiv \alpha^{*} \vee \beta^{*}$

(4) If $\phi \equiv \alpha \wedge \beta$, then $\phi^{*} \equiv \alpha^{*} \wedge \beta^{*}$

(5) If $\phi \equiv \exists x \alpha$, then $\phi^{*} \equiv \exists x_{1} x_{2} x_{3} x_{4} \alpha^{*}$

(6) If $\phi \equiv \forall x \alpha$, then $\phi^{*} \equiv \forall x_{1} x_{2} x_{3} x_{4} \alpha^{*}$.

The first big conjunct in clause (1) reflects the fact that matrices in $M_{2}\left(\mathbb{R}_{\geq 0}\right)$ have entries in $\mathbb{R}_{\geq 0}$. The second big conjunct in (1) reflects the fact that matrices in $\bar{M}_{2}\left(\mathbb{R}_{\geq 0}\right)$ are such that the first entry is in $\mathbb{R}_{>0}$. The last big conjunct in (1) states that the matrix associated with $s$ equals the matrix associated with $t$. It is obvious that $\phi$ is a theorem of $\operatorname{Th}\left(M_{2}\left(\mathbb{R}_{>0}\right)\right)$ if and only if $\phi^{*}$ is a theorem $\operatorname{Th}(\mathbb{R})$. Thus, $\operatorname{Th}\left(M_{2}\left(\mathbb{R}_{>0}\right)\right)$ is decidable.

It is not difficult to see that the model of $B T Q-B^{2} Q_{4}$ we have defined is also a model of $W D-W D_{3}$ and $W B T-W B T_{3}$. We extend it to a decidable model of $D-D_{4}$ by interpreting $\preceq$ as $M_{2}\left(\mathbb{R}_{\geq 0}\right) \times M_{2}\left(\mathbb{R}_{\geq 0}\right)$.

Our proof of decidability of $\operatorname{Th}\left(\mathrm{M}_{2}\left(\mathbb{R}_{\geq 0}\right)\right)$ is actually a 4-dimensional interpretation of $\operatorname{Th}\left(M_{2}\left(\mathbb{R}_{\geq 0}\right)\right)$ in $\operatorname{Th}(\mathbb{R})$. This means that each object in the language of $\operatorname{Th}\left(M_{2}\left(\mathbb{R}_{\geq 0}\right)\right)$ is associated with a quadruple of objects in the language of $T h(\mathbb{R})$. For more on this more general notion of interpretability, see Visser [20].

We can now state the main theorem of this section.

Theorem 8 WD, WBT and BTQ are minimal essentially undecidable.

The only thing that is lacking to show that $D$ is minimal essentially undecidable is to show that $D-D_{5}$ and $D-D_{6}$ are not essentially undecidable. A negative solution of the following problem would thus show that $D$ is minimal essentially undecidable.

Open Problem 2 Show that neither $D-D_{5}$ nor $D-D_{6}$ is essentially undecidable.

We have not put much focus on the theories BT and $C$. The proofs of Lemma 5 and Lemma 11 show that $C-C_{1}, C-C_{4}$ and $B T-B_{4}$ are not essentially undecidable. Beyond that, the minimality of BT and $\mathrm{C}$ is an open problem.

\section{Open Problem 3 Are BT and C minimal essentially undecidable?}

In this section, we investigated whether our axiom sets are minimal w.r.t set inclusion and the property of being essentially undecidable. A different notion of minimality that we have been implicitly investigating is minimality w.r.t. interpretability. We have seen that WD is interpretable in all the essentially undecidable theories we have studied. It is however not minimal in the interpretability pre-order. In [7], Jeřábek shows that there is an essentially undecidable theory that is interpretable in R but that does not interpret $\mathrm{R}$. The theory Jeřábek gives is such that all partially recursive functions are representable. In Section 3 of [2], Yong Cheng uses results in [7] to give many examples of essentially undecidable theories that are interpretable in $\mathrm{R}$ but that do not interpret R. One of the referees observed that the existence of theories strictly below R w.r.t. interpretability also follows from the work of Shoenfield [14].

Although there are many essentially undecidable theories below R w.r.t interpretability, to the best of our knowledge, it is not known whether there exists a minimal computably enumerable essentially undecidable theory, w.r.t. interpretability. For a more detailed discussion of this problem, we refer the reader to Yong Cheng [2]. It 
Fig. $3 S \rightarrow T$ means $S$ is interpretable in $T$ but $S$ does not interpret $T . S \leftrightarrow T$ means $S$ and $T$ are mutually interpretable. $S \rightarrow T$ means $S$ is interpretable in $T$ but it is unknown whether $S$ interprets $T$

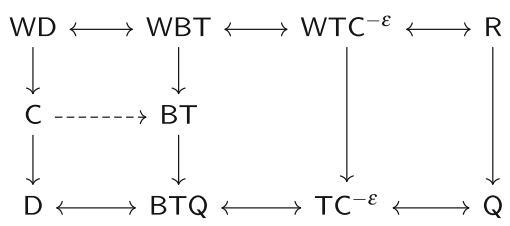

follows from the idea behind the proof of Theorem 4.7 of [2] that the interpretability degrees of computably enumerable essentially undecidable theories have infima. Hence, if there exists a minimal essentially undecidable theory, then that theory is also the minimum essentially undecidable theory.

\section{Summary}

We have formulated essentially undecidable theories WD $\subset \mathrm{C} \subset \mathrm{D}$ and WBT $\subset \mathrm{BT} \subset$ BTQ (see Fig. 3). The theories WD, WBT and R are mutually interpretable while the theories D, BTQ and Q are mutually interpretable. The theories WD, WBT and BTQ have minimal essentially undecidable axiomatizations, but it not clear whether the same is true of $D$. Both WD and $D$ are purely universally axiomatised.

Acknowledgements We thank our supervisor Lars Kristiansen for his guidance from the time we became interested in this topic to the completion of the manuscript. We thank the two anonymous referees for their insightful comments and for suggesting a proof of Lemma 9.

Funding Open access funding provided by University of Oslo (incl Oslo University Hospital).

Open Access This article is licensed under a Creative Commons Attribution 4.0 International License, which permits use, sharing, adaptation, distribution and reproduction in any medium or format, as long as you give appropriate credit to the original author(s) and the source, provide a link to the Creative Commons licence, and indicate if changes were made. The images or other third party material in this article are included in the article's Creative Commons licence, unless indicated otherwise in a credit line to the material. If material is not included in the article's Creative Commons licence and your intended use is not permitted by statutory regulation or exceeds the permitted use, you will need to obtain permission directly from the copyright holder. To view a copy of this licence, visit http://creativecommons.org/licenses/by/4.0/.

\section{References}

1. Börger, E., Grädel, E., Gurevich, Y.: The Classical Decision Problem. Universitext. Springer, Berlin Heidelberg (2001)

2. Cheng, Y.: Find the limit of incompleteness I. Bull. Symbol. Logic 26(3-4), 268-286 (2020)

3. Ganea, M.: Arithmetic on semigroups. J. Symb. Log. 74, 265-278 (2009)

4. Grzegorczyk, A.: Undecidability without arithmetization. Stud. Logica 79, 163-230 (2005)

5. Grzegorczyk, A., Zdanowski, K.: Undecidability and concatenation. In: Ehrenfeucht et al. (eds.) Andrzej Mostowski and Foundational Studies, pp. 72-91. IOS, Amsterdam (2008)

6. Higuchi, K., Horihata, Y.: Weak theories of concatenation and minimal essentially undecidable theories. Arch. Math. Logic 53, 835-853 (2014)

7. Jeřábek, E.: Recursive functions and existentially closed structures. J. Math. Logic (2020). https://doi. org/10.1142/S0219061320500026

8. Jones, J., Shepherdson, J.: Variants of Robinson's essentially undecidable theory R. Arch. Math. Logik Grundlagenforschung 23, 61-64 (1983) 
9. Kristiansen, L., Murwanashyaka, J.: Decidable and undecidable fragments of first-order concatenation theory. In: Manea, F., Miller, R., Nowotka, D. (eds.) Sailing Routes in the World of Computation. CiE 2018. Lecture Notes in Computer Science, vol. 10936. Springer, Cham (2018)

10. Kristiansen, L., Murwanashyaka, J.: First-order concatenation theory with bounded quantifiers. Arch. Math. Logic 60, 77-104 (2021). https://doi.org/10.1007/s00153-020-00735-6

11. Kristiansen, L., Murwanashyaka, J.: On Interpretability between some weak essential undecidable theories. In: Anselmo, M., Della, Vedova G., Manea, F., Pauly, A. (eds.) Beyond the Horizon of Computability. CiE 2020. Lecture Notes in Computer Science, vol. 12098. Springer, Cham (2020). https://doi.org/10.1007/978-3-030-51466-2_6

12. Murwanashyaka, J.: On First-Order Bit Theory. MA Thesis. University of Oslo (2019). http://urn.nb. no/URN:NBN:no-82577

13. Rabin, M.O.: Decidability of second-order theories and automata on infinite trees. Trans. Am. Math. Soc. 141, 1-35 (1969)

14. Shoenfield, J.R.: Degrees of formal systems. J. Symb. Logic 23(4), 389-392 (1958)

15. Smoryński, C.: Logical Number Theory I: An Introduction. Universitext. Springer, Berlin (1991)

16. Švejdar, V.: On interpretability in the theory of concatenation. Notre Dame J. Formal Logic 50, 87-95 (2009)

17. Tarski, A.: A Decision Method for Elementary Algebra and Geometry. Rand Corporation (1948)

18. Tarski, A., Mostowski, A., Robinson, R.M.: Undecidable Theories. North-Holland, Amsterdam (1953)

19. Visser, A.: Growing commas. A study of sequentiality and concatenation. Notre Dame J. Formal Logic 50, 61-85 (2009)

20. Visser, A.: Why the theory $R$ is special. In: Tennant, V (eds.) Foundational Adventures. Essays in honour of Harvey Friedman, pp. 7-23. College Publications, UK (2014)

Publisher's Note Springer Nature remains neutral with regard to jurisdictional claims in published maps and institutional affiliations. 